\title{
Wave propagation in periodic shells with tapered wall thickness and changing material properties
}

\author{
M. Toso and A. Baz* \\ Mechanical Engineering Department, University of Maryland, College Park, MD 20742, USA
}

\begin{abstract}
A theoretical method based on the Transfer Matrix Formulation and Wavelet Transforms is developed in order to effectively investigate the influence of periodicity, variable geometry and material properties on the wave propagation characteristics of axis-symmetric shells. Several experiments have been conducted to verify the numerical predictions and to demonstrate that the Wavelet Transform is a very powerful tool to uniquely identify and compare the energy distribution both in the time and frequency domain.

Thin shells are modeled as two-dimensional wave-guides, where the propagation of the longitudinal waves is coupled with the flexural (radial) waves. Variations of the wall thickness, medium radius and element length of the shell can effectively filter out/stop undesirable bands of frequencies from the longitudinal and/or the transverse wave characteristics. The principal parameter that influences the width and location of the stop bands is the ratio between the cross sections at the two ends of the shell element. Sophisticated exponential profiles and simpler linear taper are implemented and compared.

Functionally graded materials (FGM) are also investigated as an alternative way to influence the parameters of the stop bands. Combinations of the FGM and geometric taper give the flexibility needed for some very demanding applications.

Different types of periodic taper configurations have complementary effects on the wave characteristics. Combinations of these complex geometries (bi-periodic tapered cells) are presented and shown to produce the most effectual energy redistribution.
\end{abstract}

Keywords: Periodic, tapered shell, wave propagation, stop bands, wavelet

\section{Introduction}

Shells of revolution play an important role in many structural applications. Traditionally most studies have attempted to adapt the membrane model (very thin shells) for practical calculations. Generally, thick shells [3,7] present a more interlaced behavior that is not described by extended 2D models. However, the availability of faster computers has shown that analyses of solid bodies based on 3D structural models [4] yield accurate predictions of static displacements, free vibration frequencies and modes, buckling loads and mode shapes. However, many practical applications require the use of axis-symmetric shells, such as in space vehicles, aircrafts and submarine vessels. Furthermore, many of these applications rely in their operation on the use of periodic shells [13] which have unique filtering capabilities.

Several researchers have studied three-dimensional vibration of hollow circular cylinders. Early investigations were focused in applying 2D shell theory on circular cylindrical shells having continuously variable wall thickness. In 1973, Stoneking [16] formulated a set of equations to solve vibrations of clamped-clamped tapered cylinders with the partition method. In 1991, Sivadas and Ganesan presented a semi-analytical finite-element analysis for determining the natural frequencies of thin circular isotropic cylindrical shells with linear and quadratic varying

\footnotetext{
*Corresponding author. E-mail: baz@eng.umd.edu.
} 
section. In their study, Love's first approximation shell theory was considered to solve the problem and investigated different boundary conditions. In 1993, Sivadas and Ganesan improved their model by including the normal strain as well as the transverse shear effects and compared the solutions to two other approximations: the thick shell theory without normal stress and Love's model without shear and rotary inertia. Suzuki et al. [17,18] presented an analytical solution of the free vibration of a clamped-clamped circular cylindrical shell with quadratic thickness variation along the axial direction.

Basically, only three groups of researchers studied conical shells with variable thickness using 2D-based shell theory. In 1977 Penzes and Padovan [12] characterized a tapered cone with an approximate closed-form solution. Then, Irie et al. [5] used the transfer matrix approach to treat the case of free vibration of a truncated conical shell having a meridian thickness expressed by an arbitrary function. Natural frequencies and mode-shapes were numerically calculated for linear, parabolic and exponential variable thickness. Takahashi et al [19,20] wrote a series of papers on this subject. 2D models have been developed for moderately thick conical shells of variable thickness where the normal displacement component is assumed to be constant along the thickness. The tangential displacements and bending rotations is supposed to be linearly varying, as in the well-known Mindlin plate theory. In 1995, Leissa and So presented extensive studies on a 3D-based procedure to determine free vibration frequencies and modes for truncated hollow cones with arbitrary thickness by applying Ritz method. These results were refined in 1999 by Kang and Leissa.

The present paper is organized as follows: a literature survey has been presented in Section 1. In Section 2, the equations of motion are derived from the energy conservation principles using the transfer matrix approach. This approach allows further investigations of the effect of varying the geometry and/or stiffness, which are the main goals of Sections 3. Subsection 3.1 presents numerical solution of exponential and polynomial tapered shells. The propagation constants for the longitudinal and transverse waves are discussed and the time-frequency plots are generated by the Wavelet Transform for a linear profile. Subsection 3.2 investigates the possibility of using Functionally Graded Materials (FGM): the material Young's modulus is allowed to vary according to exponential or polynomial laws. Numerical solution of various examples is presented in terms of propagation constants in order to quantify the effect of geometry changes. Moreover, the combined effect of varying the geometrical profiles with the elastic properties is also investigated.

Section 4 describes the experiments conducted on a linearly tapered shell. The time response and the corresponding Wavelet Transform analysis are compared to the numerical predictions of Section 3. Section 5 extends the results of Subsection 3.1 to the case of periodic shell elements. Section 6 summarizes conclusions. Best results can be obtained when combining tapered geometry and either functionally graded materials or with periodicity. Bi-periodic tapered elements offer the most interesting behavior.

\section{Equation of motion for shells of variable material properties and geometry}

Under hypothesis of small deformation, the elastic strains $(\varepsilon, \gamma)$ for the generic three-dimensional shell shown Fig. 1 are given by [2]:

$$
\begin{aligned}
\varepsilon_{(x)} & =\frac{\partial U(x, \vartheta, r)}{\partial x}, \\
\varepsilon_{(\vartheta)} & =\frac{1}{R+r}\left[\frac{\partial V(x, \vartheta, r)}{\partial \vartheta}+W(x, \vartheta, r)\right], \\
\varepsilon_{(r)} & =\frac{\partial W(x, \vartheta, r)}{\partial r}, \\
\gamma_{(x \vartheta)} & =\frac{1}{R+r} \frac{\partial U(x, \vartheta, r)}{\partial \vartheta}+\frac{\partial V(x, \vartheta, r)}{\partial x}, \\
\gamma_{(x r)} & =\frac{\partial U(x, \vartheta, r)}{\partial r}+\frac{\partial W(x, \vartheta, r)}{\partial x}, \\
\gamma_{(x \vartheta)} & =\frac{\partial V(x, \vartheta, r)}{\partial r}+\frac{1}{R+r}\left[\frac{\partial W(x, \vartheta, r)}{\partial \vartheta}-V(x, \vartheta, r)\right],
\end{aligned}
$$




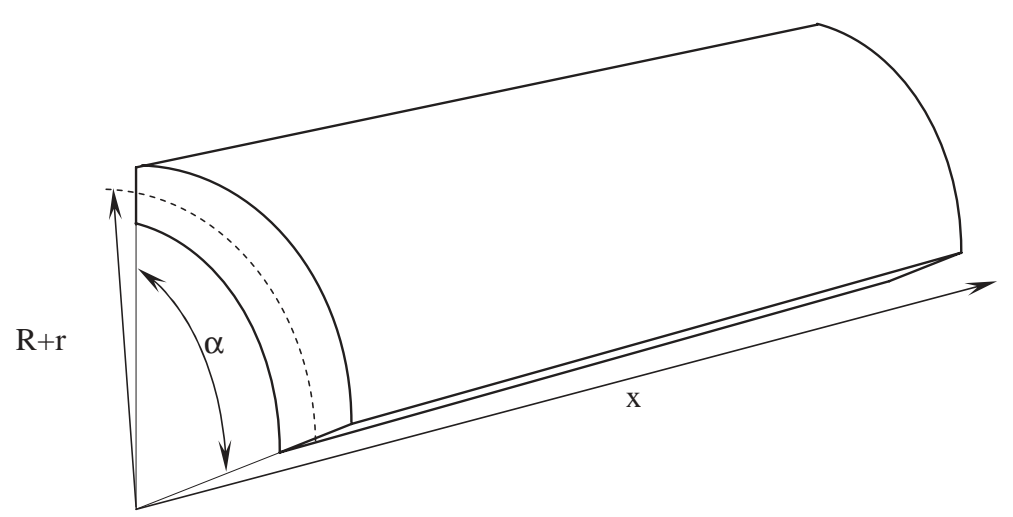

Fig. 1. Three-dimensional cylindrical shell.

where: $U, V, W$ are the displacement in the three spatial directions $(x, \vartheta, r)$ and $R$ is the medium radius.

Introducing Kirchhoff hypotheses, such that $U$ and $V$ linearly vary through the thickness and $W$ is constant through the thickness, then:

$$
\begin{aligned}
U(x, \vartheta, r) & =u(x, \vartheta)-r \frac{\partial w(x, \vartheta)}{\partial x} \\
V(x, \vartheta, r) & =v(x, \vartheta)+\frac{r}{R}\left[v(x, \vartheta)-\frac{\partial w(x, \vartheta)}{\partial \vartheta}\right], \\
W(x, \vartheta, r) & =w(x, \vartheta) .
\end{aligned}
$$

Substituting Eq. (2) into Eq. (1), a simpler expression is obtained:

$$
\begin{aligned}
\varepsilon_{(x)} & =u_{, x}-r w_{, x x}, \\
\varepsilon_{(\vartheta)} & =\frac{1}{R\left(1+\frac{r}{R}\right)} w-\frac{1}{R} v_{, \vartheta}-\frac{\frac{r}{R}}{R\left(1+\frac{r}{R}\right)} w_{, \vartheta \vartheta}, \\
\varepsilon_{(r)} & =0 \\
\gamma_{(x \vartheta)} & =\frac{1}{R\left(1+\frac{r}{R}\right)} u_{, \vartheta}-\left(1+\frac{r}{R}\right) v_{, x}-\frac{r}{R}\left[1+\frac{1}{R\left(1+\frac{r}{R}\right)}\right] w_{, x \vartheta}, \\
\gamma_{(x r)} & =-w_{, x}+w_{, x}=0, \\
\gamma_{(x \vartheta)} & =\frac{1}{R}\left(v-w_{, \vartheta}\right)+\frac{1}{R}\left(w_{, \vartheta}-v\right)=0 .
\end{aligned}
$$

If we assume the axial symmetric shell to be thin $(r / R<<1)$, and torsion and bending to be uncoupled (Donnell-Mushtari) ( $v_{, x} \cong 0 ; v_{, \vartheta} \cong 0$ ), then Eq. (3) reduce to [9]:

$$
\begin{aligned}
\varepsilon_{(x)} & =u_{, x}-r w_{, x x}, \\
\varepsilon_{(\vartheta)} & \cong \frac{1}{R} w, \\
\varepsilon_{(r)} & =0 \\
\gamma_{(x \vartheta)} & \cong 0 \\
\gamma_{(x r)}, \gamma_{(x \vartheta)} & =0 .
\end{aligned}
$$

In order to obtain the equations of motion for the axis-symmetric shell, the energy method approach is employed along with the Hamilton's principle, such that: 


$$
\int_{t 1}^{t 2} \delta(T-U+W) d t=0
$$

where $T$ is the Kinetic Energy of the structure, $U$ denotes its Potential Energy and $W$ defines the Virtual Work done by the external forces.

The general expressions of these quantities for a three-dimensional structure are:

$$
\begin{aligned}
& U=\frac{1}{2} \iiint_{V o l}\left[\sigma_{(x)} \varepsilon_{(x)}+\sigma_{(\vartheta)} \varepsilon_{(\vartheta)}+\sigma_{(r)} \varepsilon_{(r)}+\tau_{(x \vartheta)} \gamma_{(x \vartheta)}+\tau_{(r \vartheta)} \gamma_{(r \vartheta)}+\tau_{(x r)} \gamma_{(x r)}\right] d V, \\
& T=\frac{1}{2} \iiint_{V o l}\left[\dot{u}^{2}+\dot{v}^{2}+\dot{w}^{2}\right] d V
\end{aligned}
$$

$\delta W \cong 0$.

The constitutive relations for a linear elastic solid are

$$
\begin{aligned}
\sigma_{(x)} & =\frac{E}{1-v^{2}}\left(\varepsilon_{(x)}+v \varepsilon_{(\vartheta)}\right), \\
\sigma_{(\vartheta)} & =\frac{E}{1-v^{2}}\left(\varepsilon_{(\vartheta)}+v \varepsilon_{(x)}\right), \\
\sigma_{(r)} & \cong 0 .
\end{aligned}
$$

Hence, the kinetic and potential energies reduce to:

$$
\begin{aligned}
& U=\frac{1}{2} \int_{0}^{L} 2 \pi \int_{-h(x) / 2}^{h(x) / 2} \frac{E}{1-v^{2}}\left[\varepsilon_{(x)}^{2}+2 v \varepsilon_{(x)} \varepsilon_{(\vartheta)}+\epsilon_{(\vartheta)}^{2}\right] R(x) d r d x, \\
& T=\frac{1}{2} \rho \int_{0}^{L} 2 \pi R(x) h(x)\left[\dot{u}^{2}+\dot{w}^{2}\right] d x,
\end{aligned}
$$

or

$$
\begin{aligned}
& U=\frac{1}{2} \int_{0}^{L} 2 \pi\left[g\left(u_{, x}^{2}+2 v \frac{u_{, x} w}{R(x)}+\frac{w^{2}}{R(x)^{2}}\right)+q w_{, x x}^{2}\right] d x, \\
& T=\frac{1}{2} \int_{0}^{L} 2 \pi \frac{g}{c^{2}}\left[\dot{u}^{2}+\dot{w}^{2}\right] d x
\end{aligned}
$$

where:

$g=R(x) K_{t}(x)=R(x) \frac{E h(x)}{\left(1-v^{2}\right.}$ is the longitudinal rigidity of the shell,

$q=R(x) D_{t}(x)=R(x) \frac{E h(x)^{3}}{12\left(1-v^{2}\right)}$ is the bending rigidity of the shell,

and $c=\sqrt{\frac{E / \rho}{\left(1-v^{2}\right)}}$ is the characteristic wave propagation phase speed.

Applying Hamilton's principle yields the following equations of motion for the tapered shell:

$$
\left\{\begin{array}{l}
\frac{g}{c^{2}} \ddot{u}=g u_{, x x}+g_{, x} u_{, x}+v(g / R)_{, x} w+v \frac{g}{R} w_{, x} \\
\frac{g}{c^{2}} \ddot{u}+q w_{, x x x x}+2 q_{, x} w_{, x x x}+q_{, x x} w_{, x x}+\frac{g}{r^{2}} w+v \frac{g}{R} u_{, x}=0
\end{array}\right.
$$


The Separation Principle defines the displacements $u$ and $w$ in terms of:

$$
u(x, t)=u(x) e^{j \omega t} \text { and } w(x, t)=w(x) e^{j \omega t}
$$

From Eqs (10) and (11), the dynamics of the shell subjected to harmonic excitation are given by:

$$
\left\{\begin{array}{l}
u_{, x x}=-\frac{\omega^{2}}{c^{2}} u-\frac{g, x}{g} u_{, x}-v \frac{(g / R)_{, x}}{g} w-v \frac{1}{R} w_{, x} \\
w_{, x x x x}=\frac{g}{q}\left(\frac{\omega^{2}}{c^{2}}-\frac{1}{R^{2}}\right) w-\frac{q, x x}{q} w_{, x x}-2 \frac{q, x}{q} w_{, x x x}-v \frac{g}{q R} u_{, x}
\end{array}\right.
$$

It is convenient, for the further analysis, to have a state-space representation of the shell system as follows:

$$
\frac{\partial}{\partial x} U(x)=\mathbf{A}(x) U(x)
$$

where $\mathbf{A}(x)=\left[\begin{array}{cccccc}0 & 0 & 0 & 1 & 0 & 0 \\ 0 & 0 & 1 & 0 & 0 & 0 \\ 0 & 0 & 0 & 0 & 0 & 1 \\ -\frac{\omega^{2}}{c^{2}} & -v \frac{(g / R)_{, x}}{g} & -v \frac{1}{R} & -\frac{g, x}{g} & 0 & 0 \\ 0 & \frac{g}{q}\left(\frac{\omega^{2}}{c^{2}}-\frac{1}{R^{2}}\right) & 0 & -v \frac{g}{q R}-2 \frac{q, x}{q}-\frac{q, x x}{q} \\ 0 & 0 & 0 & 0 & 1 & 0\end{array}\right]$ represents the state-space matrix

and $U(x)=\left\{\begin{array}{llllll}u & w & w_{, x} & u_{, x} & w_{, x x} & w_{, x x x}\end{array}\right\}^{T}$ is the state-space vector.

Solutions to Eq. (13) are calculated by the integration over the shell length $\mathrm{L}$ to give:

$$
U(L)=e^{\left[\int_{0}^{L}[A(x)] d x\right]} U(0)
$$

The last three components of the state vector $U(x)$ can be transformed into generalized forces (traction $N$, shear $Q$, bending moment $M$ ) through the coordinate transformation matrix $\left[G_{x}\right]$ and a new state vector $Y$ is obtained:

$$
Y(x)=\mathbf{G}_{x} U(x)
$$

where

$$
Y(x)=\left\{\begin{array}{llll}
u & w & w_{, x} & N Q M
\end{array}\right\}^{T}=\text { transfer vector at } x
$$

and

$$
\mathbf{G}_{x}=\left[\begin{array}{cccccc}
1 & 0 & 0 & 0 & 0 & 0 \\
0 & 1 & 0 & 0 & 0 & 0 \\
0 & 0 & 1 & 0 & 0 & 0 \\
0 & \frac{v}{R(x)} K_{t}(x) & 0 & K_{t}(x) & 0 & 0 \\
0 & 0 & 0 & 0 & -D_{t}(x) & 0 \\
0 & 0 & 0 & 0 & 0 & D_{t}(x)
\end{array}\right] \triangleq=\text { transformation matrix }
$$

The vector $Y$ in Eq. transforms Eq. (14) into the Transfer Matrix representation as follows:

$$
Y_{L}=\left[\mathbf{G}_{L} e^{\int_{0}^{L} A(x) d x} \mathbf{G}_{0}^{-1}\right] Y_{0}=\mathbf{T} Y_{0}
$$

where $(x=0) \Rightarrow Y_{0}=\left[\mathbf{G}_{0}\right] U(0)$ and $(x=L) \Rightarrow Y_{L}=\left[\mathbf{G}_{L}\right] U(L)$.

The eigenvalues $\lambda_{i}$ of the transfer matrix $T$ give all the information about the propagation characteristics:

$$
\lambda_{i}=e^{\mu_{i}}=e^{\alpha_{i}} e^{j \beta_{i}}
$$


Table 1

PVC Shell Properties

\begin{tabular}{lc}
\hline Parameter & Value \\
\hline Young Modulus $[\mathrm{Pa}]$ & $3.610^{9}$ \\
Density $\left[\mathrm{Kg} / \mathrm{m}^{3}\right]$ & 1700 \\
Length $[\mathrm{mm}]$ & $315\left(123 / 8^{\prime \prime}\right)$ \\
External Diameter $[\mathrm{mm}]$ & $48\left(1.9^{\prime \prime}\right)$ \\
Internal Diameter $[\mathrm{mm}]$ & $40\left(1.58^{\prime \prime}\right)$ \\
Geometric Taper Ratio & 4 \\
FGM Ratio & 10 \\
\hline
\end{tabular}

where $\mu_{i}$ are the propagation parameter, and $\alpha_{i}$ and $\beta_{i}$ are called attenuation factor and phase angle and represent the real and imaginary portion of the propagation constant.

By recalling equations (16) and (17), each wave component can be written in indicial notation as:

$$
\left(Y_{L}\right)_{i}=e^{\alpha_{i}}\left(Y_{O}\right)_{i} e^{j \beta_{i}}
$$

Equation (18) shows that the disturbance measured at location $L$ is shifted in phase by the factor $\beta$ (phase angle) with respect to the signal measured at location 0 and it is magnified or attenuated by factor $\alpha$ (attenuation factor) depending on the taper ratio. When the phase $\beta$ shifts to $180^{\circ}$, destructive interference occurs between the traveling wave and the reflected wave so that no remaining disturbance propagates along the shell.

It is important here to note that Young's modulus $E$ of the shell is replaced by the complex modulus $E=E^{\prime}(1+j \eta)$ with $E^{\prime}$ and $\eta$ denoting the storage modulus and loss factor of the material. Also, the computation of the exponential matrix $e^{\left[\int_{0}^{L}[A(x)] d x\right]}$, in Eqs (14) and (16) is carried out using the scaling and squaring algorithm of MATLAB with a Pade approximation to ensure efficient convergence.

\section{Numerical examples}

A plastic composite shell is considered in this study. The basic properties of this shell are listed in Table (1). Changes in the material properties and/or geometry can affect considerably the propagation characteristics of the structure.

\subsection{Geometrically tapered profiles}

It is of great interest to study the influence of thickness variation along the length of a straight cylindrical shell. In the present study, only the internal diameter will be profiled, while the outer diameter is maintained constant.

Hence, the medium radius $R(x)$ at any cross-section $x$ is represented as function of the fixed outer diameter $D_{0}$ and the variable thickness $h(x)$ as follows:

$$
R(x)=\frac{D_{0}-h(x)}{2}
$$

In order to compare results with the characteristics of a typical uniform shell, the mass is also kept constant, which adds a constraint on the initial thickness $h_{0}$, such as

$$
\int_{\text {Vol }} R(x) h(x) d V=\pi\left(D_{0}-h_{u}\right) h_{u} L
$$

Two different profiles are presented in comparison with the uniform shell, namely the exponential profile and the polynomial profile. 


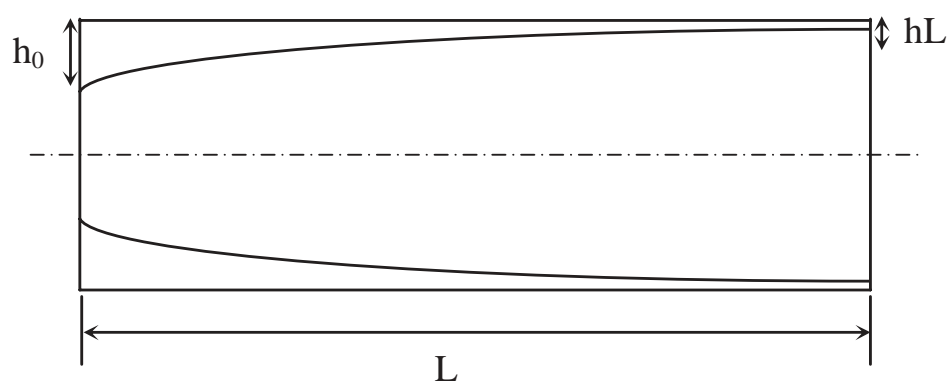

Fig. 2. Cross-section of exponential-tapered shell.

\subsubsection{Exponential profile}

The thickness $h(x)$ is modeled as follows, according to Fig. 2:

$$
h(x)=h_{0} e^{a \frac{x}{L}}
$$

Parameter a is quickly identified when the thickness ratio is decided:

$$
a=\ln \frac{h_{L}}{h_{0}}
$$

The eigenvalues problem defined by Eqs (16) and (17) is numerically solved with Matlab.

The expression for the state-space matrix $\mathbf{A}$ and the transformation matrix $\mathbf{G}_{x}$ reduce to:

$$
[\mathbf{A}(x)]=\left[\begin{array}{cccccc}
0 & 0 & 0 & 1 & 0 & 0 \\
0 & 0 & 1 & 0 & 0 & 0 \\
0 & 0 & 0 & 0 & 0 & 1 \\
-\frac{\omega^{2}}{c^{2}} & -\left(\frac{a}{L}\right)\left(\frac{v}{R(x)}\right) & -\frac{v}{R(x)}-\left(\frac{a}{L}\right)\left(1-\frac{h(x)}{2 R(x)}\right) & 0 & 0 \\
0\left(\frac{12}{h^{2}(x)}\right)\left(\frac{\omega^{2}}{c^{2}}-\frac{1}{R^{2}(x)}\right) & 0 & 0 & -v\left(\frac{12}{R(x) h^{2}(x)}\right) & -2\left(\frac{a}{L}\right)\left(3-\frac{h(x)}{2 R(x)}\right)-\left(\frac{a}{L}\right)^{2} & \left(9-7 \frac{h(x)}{2 R(x)}\right) \\
0 & 0 & 0 & 1 & 0
\end{array}\right]
$$

and

$$
\left[\mathbf{G}_{x}\right]=\left[\begin{array}{cccccc}
1 & 0 & 0 & 0 & 0 & 0 \\
0 & 1 & 0 & 0 & 0 & 0 \\
0 & 0 & 1 & 0 & 0 & 0 \\
0 & \frac{v E h(x)}{\left(1-v^{2}\right) R(x)} & 0 & \frac{E h(x)}{\left(1-v^{2}\right)} & 0 & 0 \\
0 & 0 & 0 & 0 & -\frac{E h(x)^{3} R(x)}{12\left(1-v^{2}\right)} & 0 \\
0 & 0 & 0 & 0 & 0 & \frac{E h(x)^{3} R(x)}{12\left(1-v^{2}\right)}
\end{array}\right]
$$

The propagation constants are plotted in the frequency domain (Fig. 3), where one can recognize the longitudinal and the bending characteristics and compare them to the corresponding characteristics of a uniformly shaped cylinder:

\subsubsection{Polynomial profile}

The thickness $h(x)$ according to Fig. 4, is given by:

$$
h(x)=h_{0}\left(1+a \frac{x}{L}\right)^{m}
$$

where the parameter a is function of the thickness ratio:

$$
a=\sqrt[m]{\frac{h_{L}}{h_{0}}}-1
$$



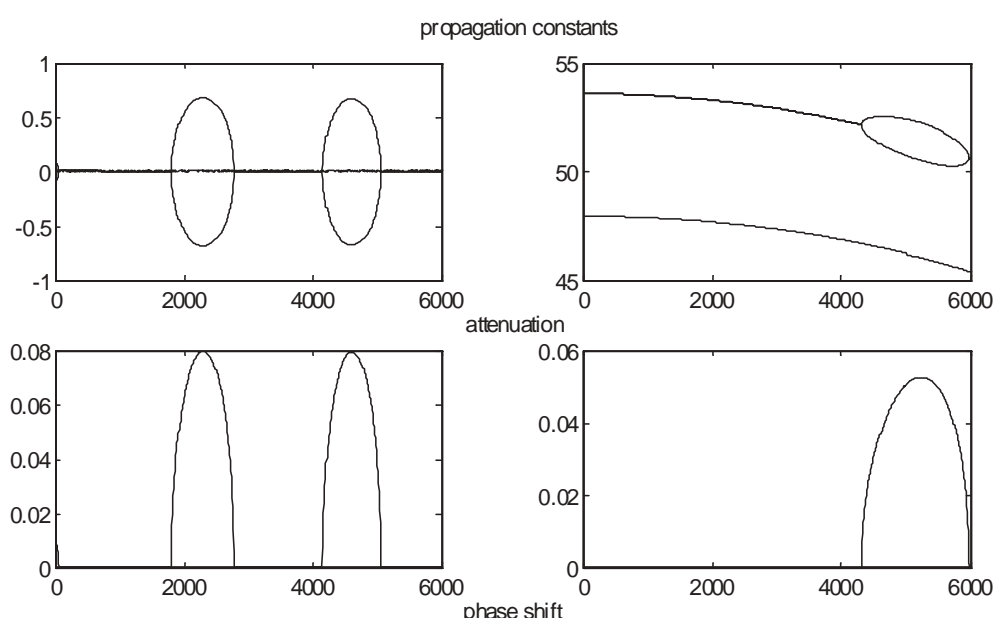

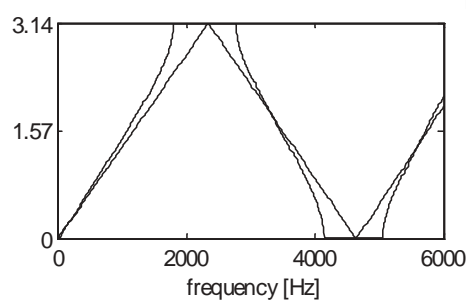

(a)

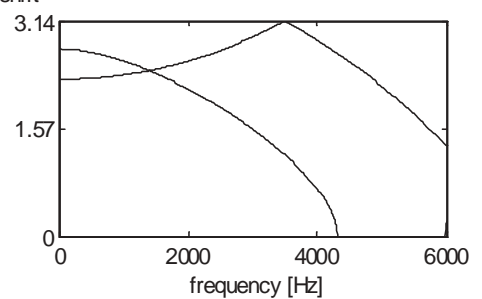

(b)

Fig. 3. Propagation constants of uniform (dash) and exponential-tapered (solid) shell: (a) longitudinal direction, (b) radial direction.

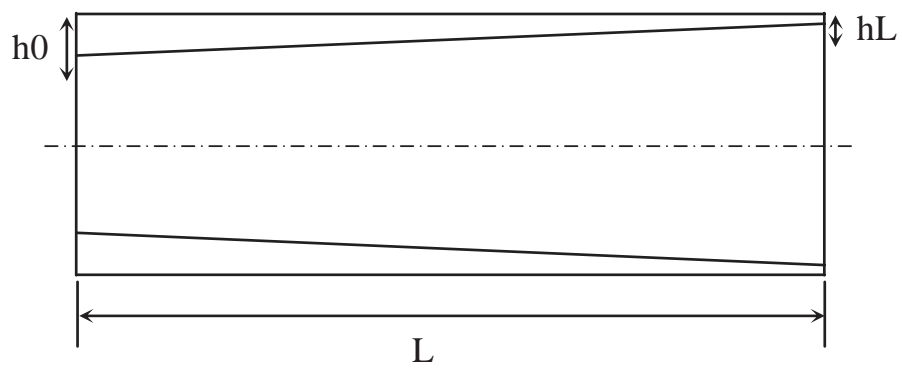

Fig. 4. Cross-section of a linear-tapered shell.

Hence, the state-space matrix $\mathbf{A}$ and the transformation matrix $\mathbf{G}_{x}$ become:

$$
\begin{aligned}
& {[\mathbf{A}(x)]=} \\
& {\left[\begin{array}{cccccc}
0 & 0 & 0 & 1 & 0 & 0 \\
0 & 0 & 1 & 0 & 0 & 0 \\
0 & 0 & 0 & 0 & 0 & 1 \\
-\frac{\omega^{2}}{c^{2}}-v\left(\frac{a}{L}\right)\left(\frac{1}{h(x)\left(D_{0}-h(x)\right)}\right) & -v\left(\frac{2}{D_{0}-h(x)}\right)-\left(\frac{a}{L}\right)\left(\frac{D_{0}-2 h(x)}{h(x)\left(D_{0}-h(x)\right)}\right) & 0 & 0 \\
0 & \left(\frac{12}{h^{2}(x)}\right)\left(\frac{\omega^{2}}{c^{2}}-\frac{4}{\left.D_{0}-h(x)\right)^{2}}\right) & 0 & -v\left(\frac{12}{h^{2}(x)}\right) & -2\left(\frac{a}{L}\right) & -6\left(\frac{a}{L}\right)^{2} \\
0 & 0 & 0 & \left(\frac{2}{\left.D_{0}-h(x)\right)}\right) & \left(\frac{3 D_{0}-4 h(x)}{h(x)\left(D_{0}-h(x)\right)}\right)\left(\frac{D_{0}-2 h(x)}{h(x)^{2}\left(D_{0}-h(x)\right)}\right) \\
0 & 0 & 0 & 1
\end{array}\right]}
\end{aligned}
$$

and 


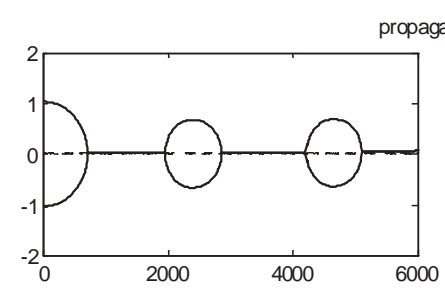

ropagation constants

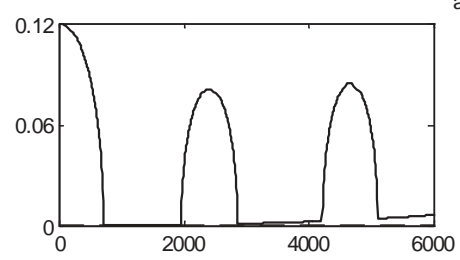
attenuation
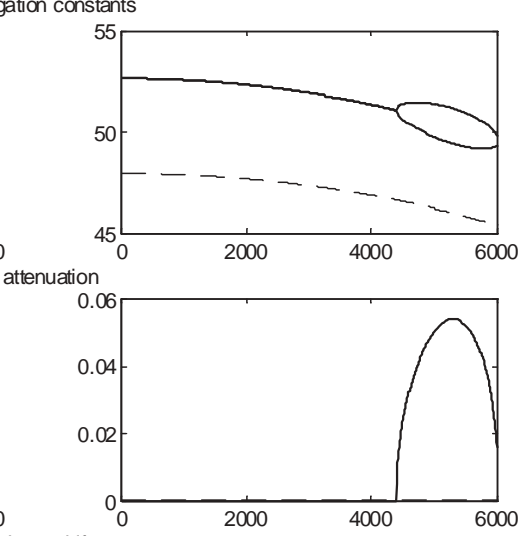

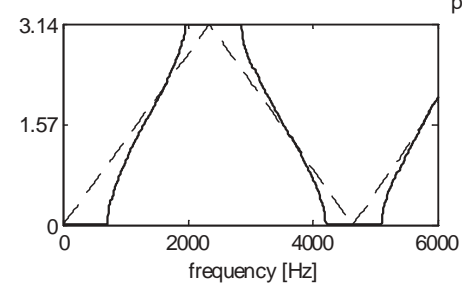

(a)

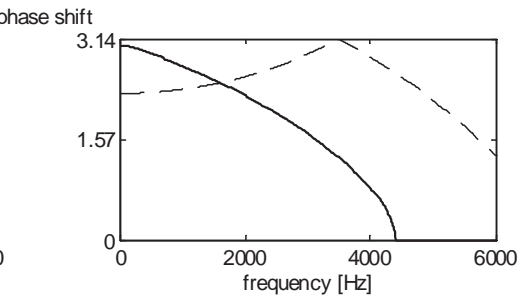

(b)

Fig. 5. Propagation constants of uniform (dash) and linear-tapered (solid) shell: (a) longitudinal direction, (b) radial direction.

$$
\left[\mathbf{G}_{x}\right]=\left[\begin{array}{cccccc}
1 & 0 & 0 & 0 & 0 & 0 \\
0 & 1 & 0 & 0 & 0 & 0 \\
0 & 0 & 1 & 0 & 0 & 0 \\
0 & \frac{2 v E h(x)}{\left(1-v^{2}\right)\left(D_{0}-h(x)\right)} & 0 & \frac{E h(x)}{\left(1-v^{2}\right)} & 0 & 0 \\
0 & 0 & 0 & 0 & -\frac{E h(x)^{3}\left(D_{0}-h(x)\right)}{24\left(1-v^{2}\right)} & 0 \\
0 & 0 & 0 & 0 & 0 & \frac{E h(x)^{3}\left(D_{0}-h(x)\right)}{24\left(1-v^{2}\right)}
\end{array}\right]
$$

Emphasis is placed here on linearly tapered shells $(m=1)$ because of the ease of their machining. Figure 5 shows the propagation constants in the frequency domain. Such characteristics can be compared to that of shells with exponential taper (Fig. 3). The interesting point is that there is no significant difference in the performance between the two profiles. Indeed, for the actual choice of dimensions and material, the longitudinal attenuation is more effective at low frequencies than the case of exponential profile. The radial propagation characteristics are comparable in the two cases.

In order to gain a better understanding of the energy distribution of the shell in the time and frequency domains, the Wavelet transform technique is applied when the shell is subjected to an impulsive load exerted at location 0 . Figure 6 emphasizes that the transmitted energy shifts to higher modes and the peak below $1 \mathrm{kHz}$ vanishes.

\subsection{Functionally graded materials (FGM)}

The other point of interest is tuning Young's modulus of the material in order to improve the vibration properties.

First, comparisons are made with plain shell, and then the benefit of combining the effect of geometrical changes with FGM will be considered. Young's Modulus is modeled as either a polynomial function or an exponential function, following the same line as the previous chapter.

\subsubsection{FGM effect: Exponential grading}

The exponential model of Young's modulus writes as: 

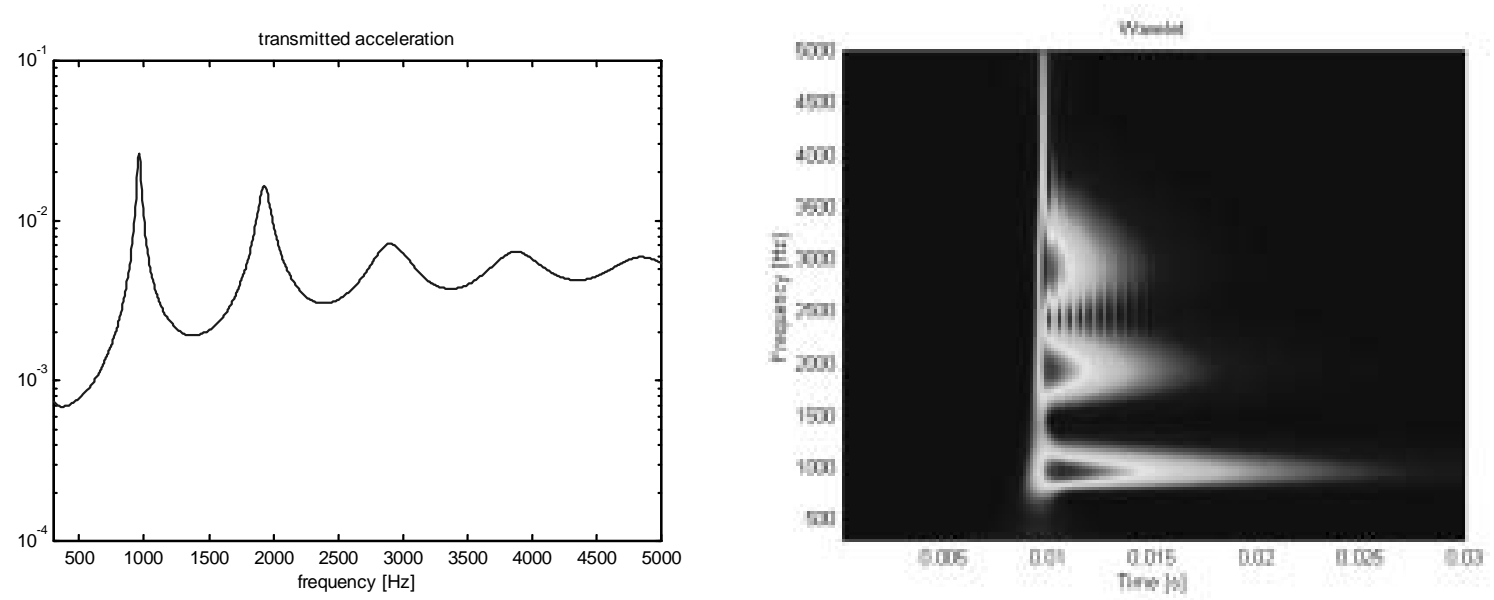

(a)
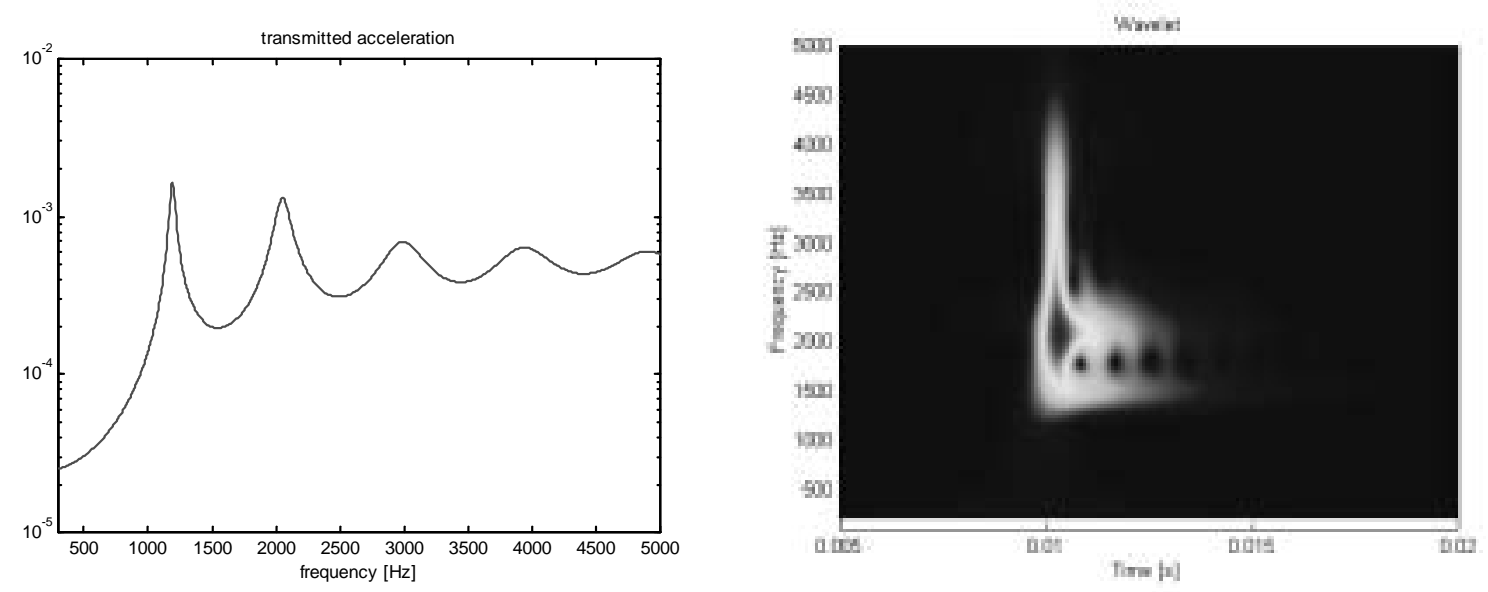

(b)

Fig. 6. Numerical frequency response and Wavelet Transform. (a) uniform thickness, (b) tapered profile.

$$
E(x)=E_{0} e^{e \frac{x}{L}}
$$

where the coefficient $e$ is more conveniently calculated from the modulus ratio between the two ends of the shell:

$$
e=\ln \frac{E_{L}}{E_{0}}
$$

Recalling the general solution for the equations of motion obtained in Section 2, Equations (12) to (17), the following expression for the state-space matrix $\mathbf{A}$ are obtained:

$$
[\mathbf{A}(x)]=\left[\begin{array}{cccccc}
0 & 0 & 0 & 1 & 0 & 0 \\
0 & 0 & 1 & 0 & 0 & 0 \\
0 & 0 & 0 & 0 & 0 & 1 \\
-\frac{\omega^{2}}{c^{2}} & -\left(\frac{e}{L}\right) \frac{v}{R} & -\frac{v}{R} & -\left(\frac{e}{L}\right) & 0 & 0 \\
0 & \left(\frac{12}{h^{2}}\right)\left(\frac{\omega^{2}}{c^{2}}-\frac{1}{R^{2}}\right) & 0 & -\frac{12 v}{R h^{2}} & -2\left(\frac{e}{L}\right) & -\left(\frac{e}{L}\right)^{2} \\
0 & 0 & 0 & 0 & 1 & 0
\end{array}\right]
$$

Also, the transformation matrix $\mathbf{G}_{x}$ has the following form: 
propagation parameter
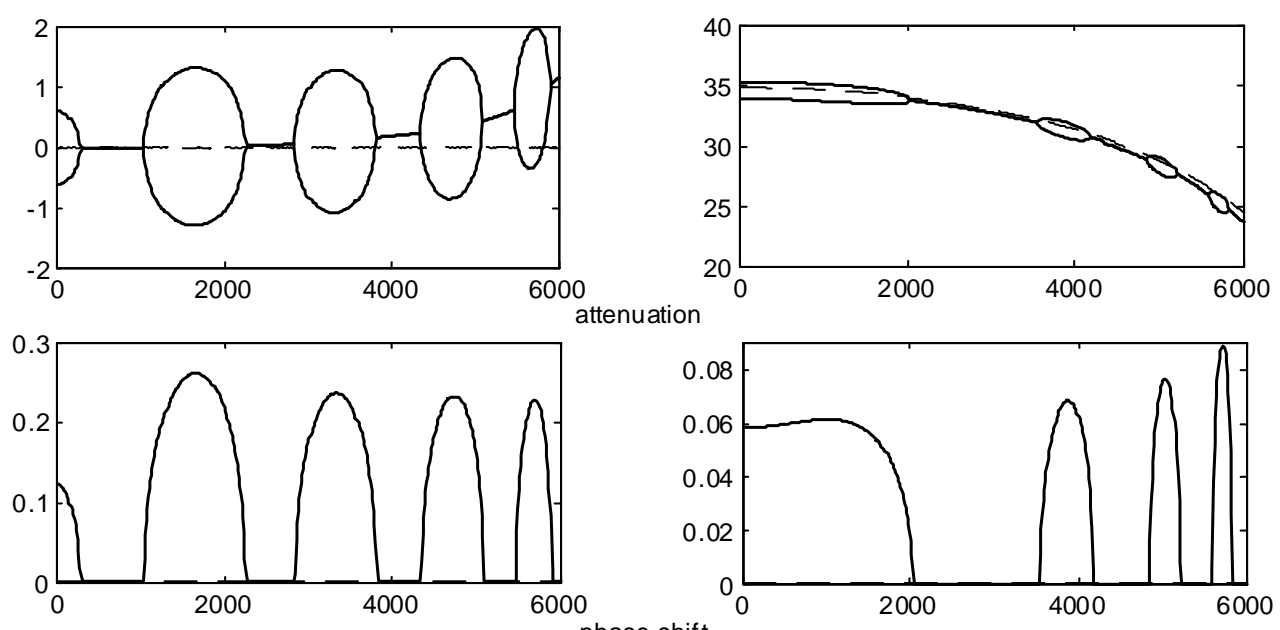

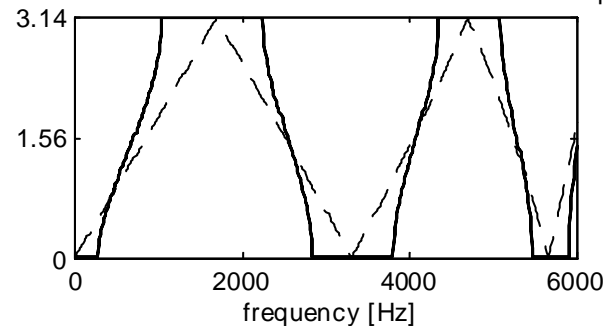

(a)

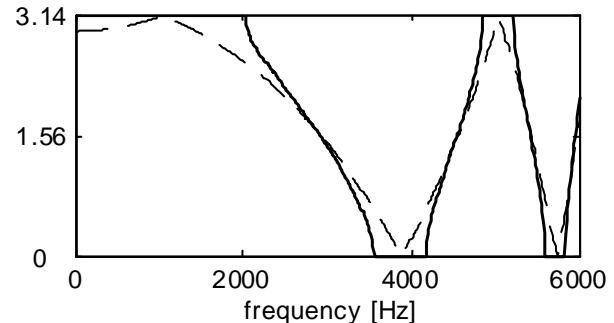

(b)

Fig. 7. Propagation constants of uniform (dash) and exponential FGM (solid) shells: (a) longitudinal direction, (b) radial direction.

$$
\left[\mathbf{G}_{x}\right]=\left[\begin{array}{cccccc}
1 & 0 & 0 & 0 & 0 & 0 \\
0 & 1 & 0 & 0 & 0 & 0 \\
0 & 0 & 1 & 0 & 0 & 0 \\
0 & \frac{v E(x)}{\left(1-v^{2}\right) R} h & 0 & \frac{E(x)}{\left(1-v^{2}\right)} h & 0 & 0 \\
0 & 0 & 0 & 0 & -\frac{E(x)}{12\left(1-v^{2}\right)} R h^{3} & 0 \\
0 & 0 & 0 & 0 & 0 & \frac{E(x)}{12\left(1-v^{2}\right)} R h^{3}
\end{array}\right]
$$

The propagation constants of the FGM shell (solid lines) are compared to the uniform shell (dashed lines) in Fig. 7 in the case when the Young modulus ratio is 10. These characteristics show a principal cut-off frequency at about $2000 \mathrm{~Hz}$ for the longitudinal and radial wave characteristics. Other interesting regions of interference (Stop Band) occur around $4 \mathrm{kHz}$ and $5.2 \mathrm{kHz}$.

\subsubsection{FGM effect: Linear grading}

For a linear Young's modulus we have:

$$
E(x)=E_{0}\left(1+e \frac{x}{L}\right)
$$

The parameter e is depends on the modulus ratio:

$$
e=\sqrt{\frac{E_{L}}{E_{0}}}-1
$$

Hence, the general expression for the state-space matrix $\mathbf{A}$ is: 

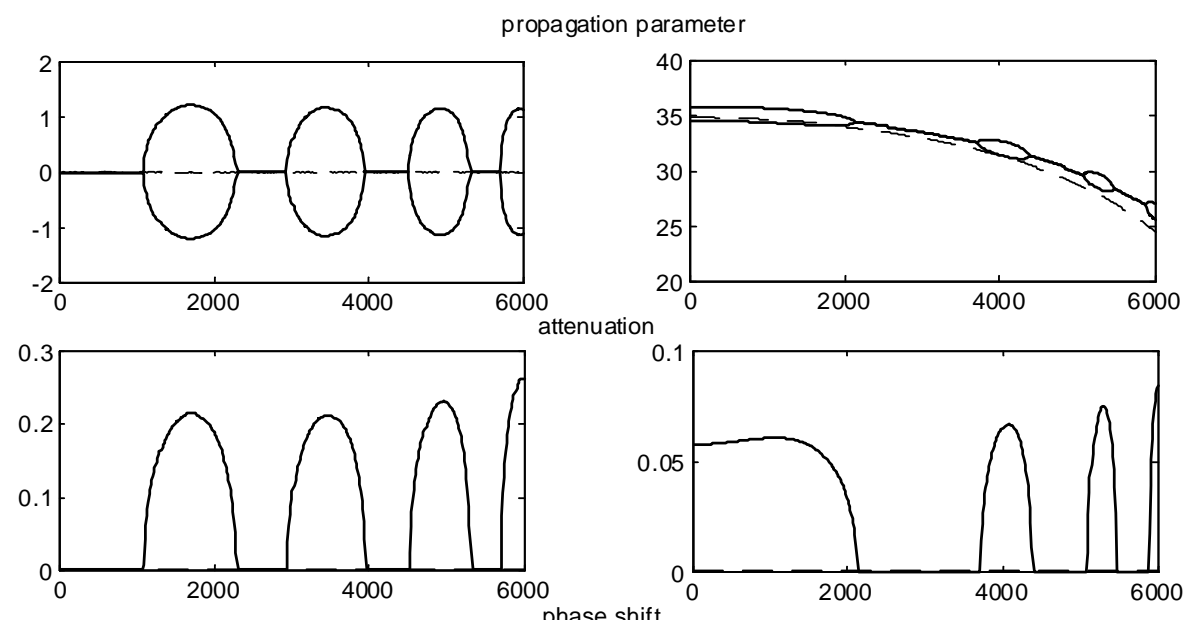

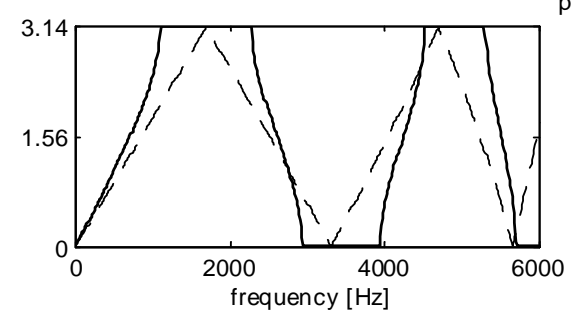

(a)

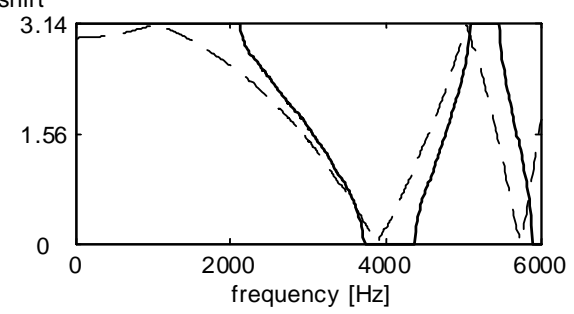

(b)

Fig. 8. Propagation constants of uniform (dash) and linear FGM (solid) shells (a) longitudinal direction, (b) radial direction.

$$
[\mathbf{A}(x)]=\left[\begin{array}{cccccc}
0 & 0 & 0 & 1 & 0 & 0 \\
0 & 0 & 1 & 0 & 0 & 0 \\
0 & 0 & 0 & 0 & 0 & 1 \\
-\frac{\omega^{2}}{c^{2}} & -\frac{\left(\frac{e}{L}\right)}{\left(1+e \frac{x}{L}\right)} \frac{v}{R} & -\frac{v}{R} & -\frac{\left(\frac{e}{L}\right)}{\left(1+\frac{x}{L}\right)} & 0 & 0 \\
0 & \left(\frac{12}{h^{2}}\right)\left(\frac{\omega^{2}}{c^{2}}-\frac{1}{R^{2}}\right) & 0 & -\frac{12 v}{R h^{2}} & -2 \frac{\left(\frac{e}{L}\right)}{\left(1+e \frac{x}{L}\right)} & 0 \\
0 & 0 & 0 & 0 & 1 & 0
\end{array}\right]
$$

where we notice the main difference with Eq. (31) is that the term $\mathrm{A}[5,6]=0$ because the second derivative of the linear profile vanishes.

The propagation constants for the shell of Table (1) can be plotted in the frequency domain, as shown in Fig. 8. The characteristics are very similar to those obtained for the exponentially graded shell (Fig. 7). More precisely, the cut-off frequency at about $2000 \mathrm{~Hz}$ in the radial direction is still present (solid line) as well as the other stop bands at $4 \mathrm{kHz}$ and $5 \mathrm{kHz}$.

\subsection{Combined effect}

Lastly, one can combine the effect of geometric taper with the functionally graded Young's modulus in order to have more control over the propagation characteristic of the shell. The resulting system matrix $[\mathbf{A}]$ is:

$$
[\mathbf{A}(x)]=\left[\begin{array}{cccccc}
0 & 0 & 0 & 1 & 0 & 0 \\
0 & 0 & 1 & 0 & 0 & 0 \\
0 & 0 & 0 & 0 & 0 & 1 \\
-\frac{\omega^{2}}{c^{2}} & -v A_{42} & -v A_{43} & -A_{44} & 0 & 0 \\
0 & A_{52 a} \frac{\omega^{2}}{c^{2}}-A_{52 b} & 0 & -v A_{54} & -2 A_{55} & -A_{56} \\
0 & 0 & 0 & 0 & 1 & 0
\end{array}\right]
$$



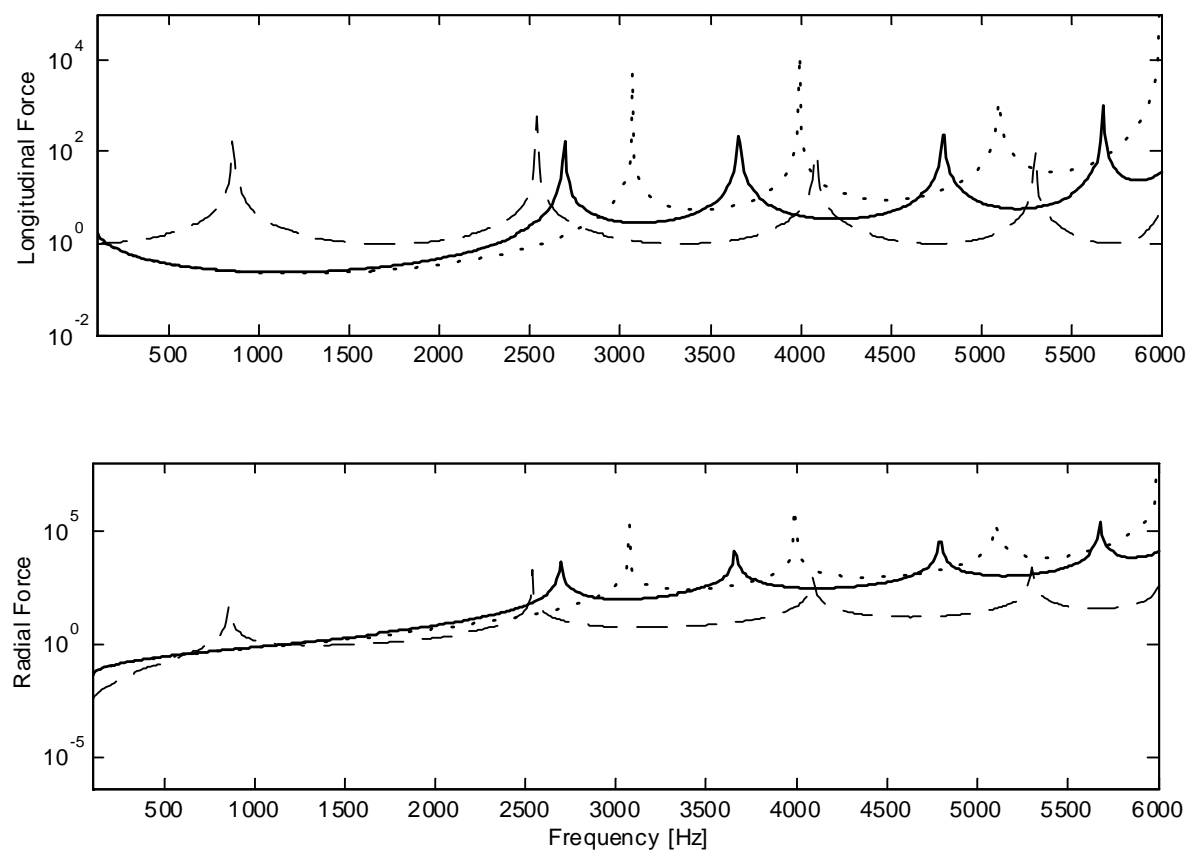

Fig. 9. Frequency response of linearly tapered FGM shells: (dash) uniform thickness, (dot) same slope, (solid) opposite slope.

where

$$
\begin{aligned}
A_{42} & =\frac{(g / R)_{x}}{g}=\frac{E_{x}}{E R}+\frac{h_{x}}{R h}, \\
A_{43} & =\frac{1}{R}=\frac{2}{D_{0}-h}, \\
A_{44} & =\frac{g_{x}}{g}=\frac{E_{x}}{E}+\frac{R_{x}}{R}+\frac{h_{x}}{h}, \\
A_{52 a} & =\frac{g}{q}=\frac{12}{h^{2}}, \\
A_{52 b} & =\frac{g}{q R}=\frac{12}{R h^{2}}, \\
A_{54} & =\frac{g}{q R^{2}}=\frac{12}{(R h)^{2}}, \\
A_{55} & =\frac{q_{x}}{q}=\frac{E_{x}}{E}+\frac{R_{x}}{R}+3 \frac{h_{x}}{h}, \\
A_{56} & =\frac{q_{x x}}{q}=\frac{E_{x x}}{E}+2 \frac{E_{x}}{E} \frac{\left(R h^{3}\right)_{x}}{R h^{3}}+\frac{\left(R h^{3}\right)_{x x}}{R h^{3}} .
\end{aligned}
$$

A numerical comparison between the three different possibilities has been carried out and obtained results are displayed in Fig. 9. In all the characteristics, the Young's modulus is assumed to be linearly decreasing. The dashed line denotes the response of a shell with uniform thickness, where as the dotted line defines the performance of a shell with linearly decreasing thickness and the solid line is corresponding to shells with linearly increasing thickness.

As expected, the combined effect of decreasing Young's modulus and decreasing thickness gives the largest stop band. As a result of this analysis we can conclude that it is possible to effectively control the spectral location and 


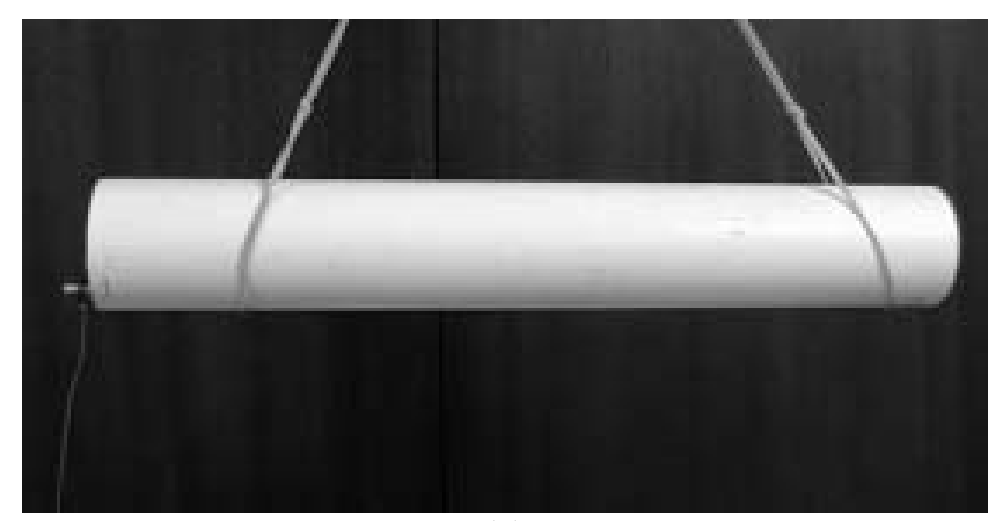

(a)

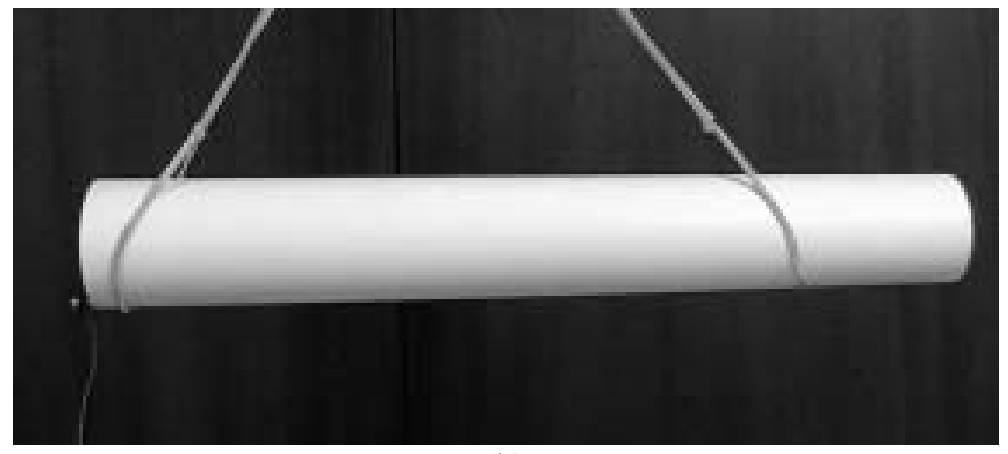

(b)

Fig. 10. Experiment: (a) uniform PVC shell, (b) tapered PVC shell.

width of the stop bands over a wider range of frequency spectrum, with proper selection of geometrical and material parameters.

\section{Experimental characteristics of shells with varying geometry}

A series of experiments are carried out in order to validate the numerical results presented in Section 3. Manufacturing considerations suggested keeping the internal diameter constant and letting the outer profile be linearly varying. Dimensions and material properties of the shell used in the experiments have been summarized in Table (1). One of the two Polyvinyl Chloride (PVC) shells has uniform profile while the other is tapered with a geometric ratio $A_{L} / A_{0}=4$. Figure 10 shows photographs of the shells used.

An impact hammer (PCB Model 086C02) is used to exert a longitudinal impulsive excitation on one end of the shell. The longitudinal acceleration at the shell end opposite to the force location is captured by a piezoelectric accelerometer (PCB Model 303A03). The spectrum analyzer (ONO SOKKI Model CF910) is triggered by the input force. The analyzer is used to record the signal coming from the accelerometer for 30 milliseconds. The stored transient response is analyzed through the Wavelet Transform (WT) in order to capture the energy content associated with the propagation spectrum. The WT allows for displaying the energy distribution simultaneously in the time and frequency domain and hence allows for fully describing the filtering characteristics of the considered classes of shells. Results are displayed in Fig. 11.

Figure 11(a) shows that the uniform profile propagates sinusoidal waves with no dispersion. The energy looks equally distributed about the two most significant peaks at approximately $900 \mathrm{~Hz}$ and $2500 \mathrm{~Hz}$. On the other hand, the tapered profile, pictured in Fig. 11(b), deforms the impulsive wave as time progresses. This reflects the shift in frequency of the energy content represented by the WT plot. Also, most of the energy is concentrated about the $2500 \mathrm{~Hz}$ peak and the peak at $900 \mathrm{~Hz}$ has been significantly cut off. These results confirm the prediction obtained by the numerical model displayed in Fig. 6 . 
Table 2

Periodic Tapered Elements

\begin{tabular}{cc}
\hline Single sub-element \\
Type(A)
\end{tabular}
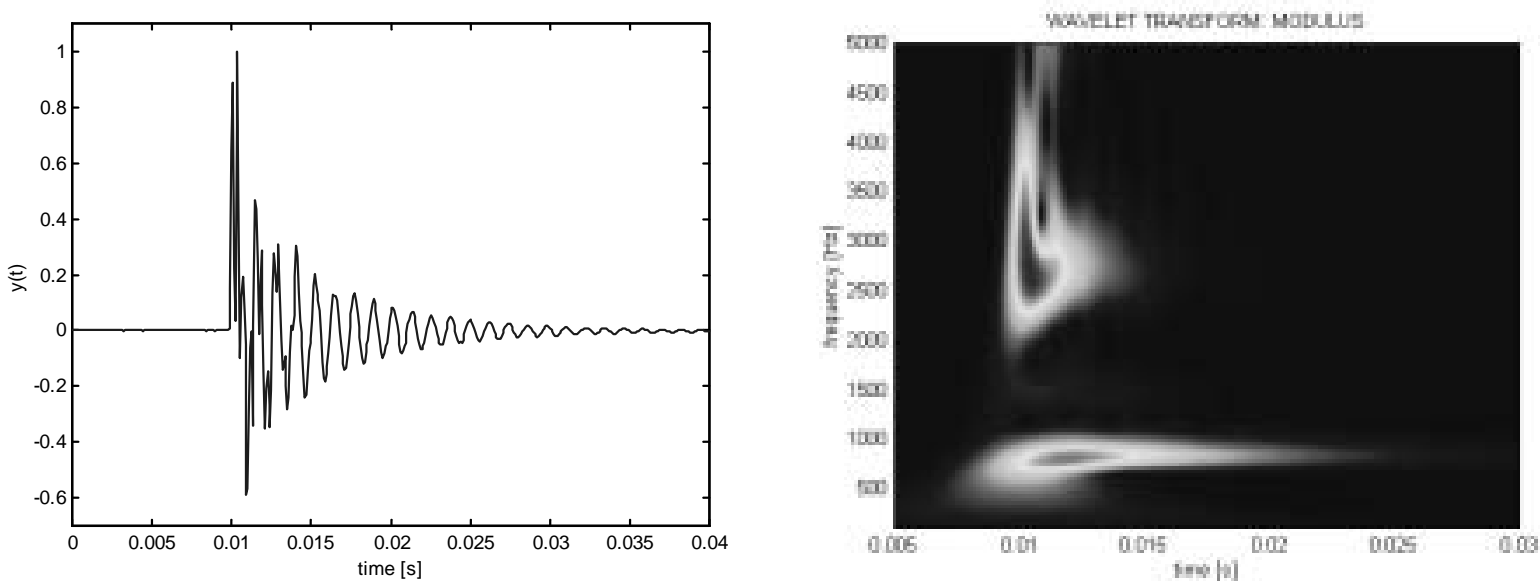

(a)
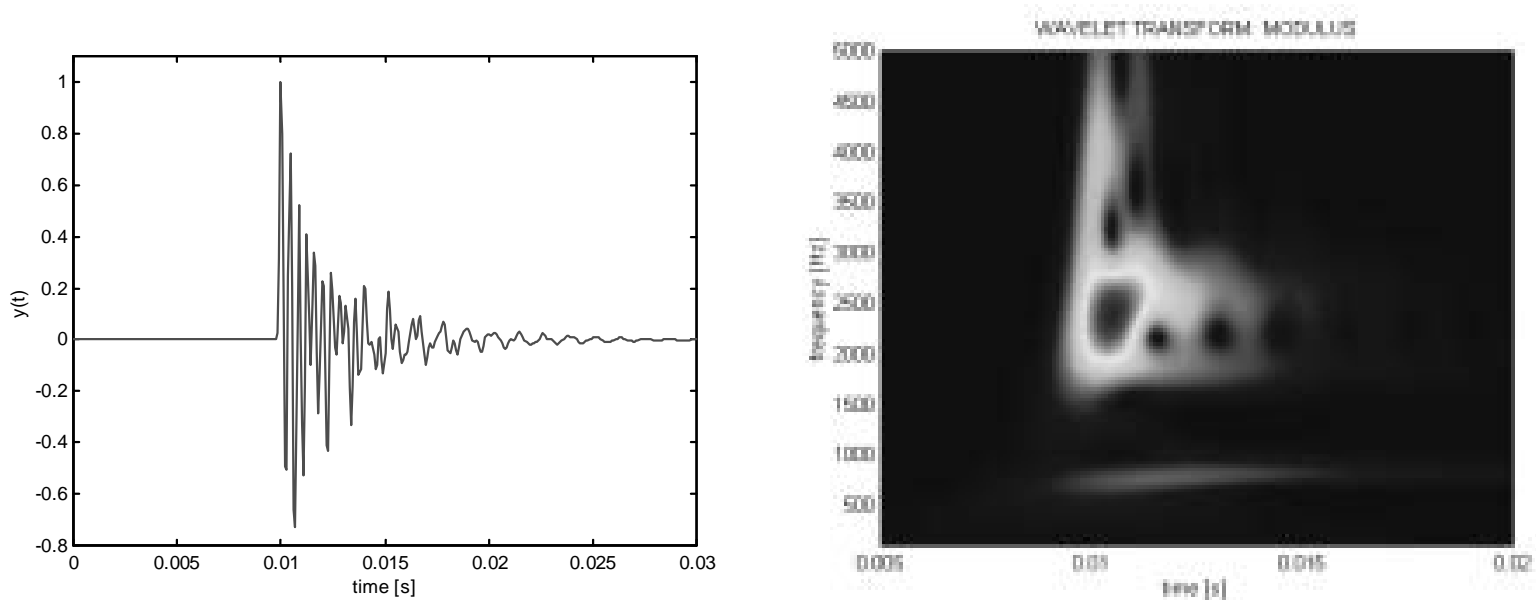

(b)

Fig. 11. Experimental Time Response and Wavelet Transform: (a) uniform thickness, (b) tapered profile.

\section{Periodic shells with tapered elements}

Further improvements in the wave propagation characteristics can be obtained by connecting a series of tapered shell elements. It is proved that elements which length is comparable to the diameter perform better than very long elements. Therefore, being the medium radius $0.87 \mathrm{in}$. (Table 1), the length of the tapered element is limited to about 2 inches.

Three different configurations are investigated and each of them brings different improvements. Table 2 shows the different configurations. 

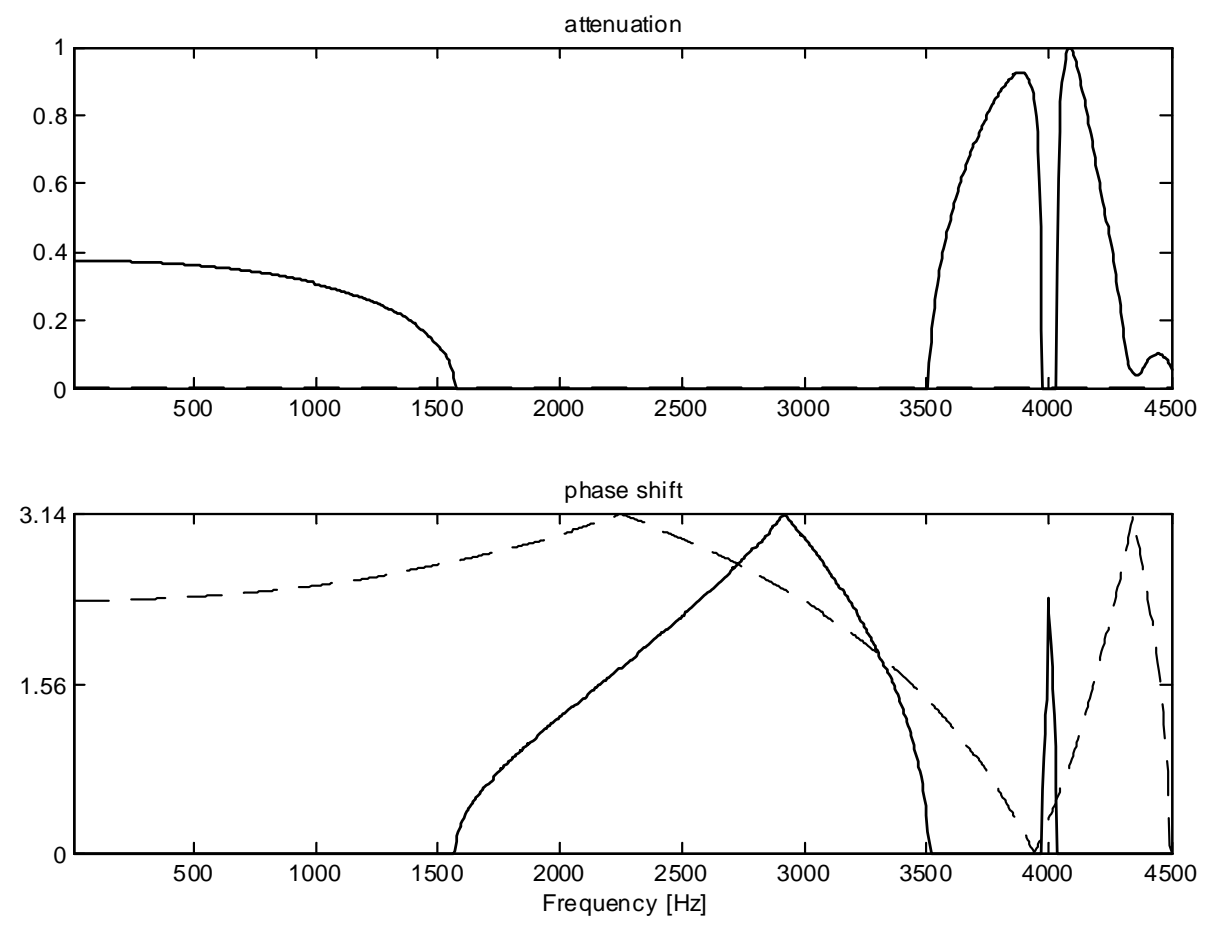

Fig. 12. Propagation characteristics of a periodic tapered shell type (A).

The characteristics of configuration (A) have already been discussed in details in Section 3.1. The shorter length of the element amplifies the width of the stop bands while periodicity amplifies the amount of the energy redistribution. Figure 12 represents the characteristics of the tapered element with solid lines and the uniform characteristics in dashed. The principal cut-off frequency has increased to about $1.5 \mathrm{kHz}$. Another stop band is located at about $3.6 \mathrm{kHz}$. Figure 13 displays the overall effect on the frequency response of a periodic shell with 4 consecutive cells.

Configuration (B) consists of two tapered sub-elements of opposite taper ratio. Although it appears to be less effective than type (A) at lower frequencies, the characteristics portrayed in Fig. 14 show the principal stop band around $2.5 \mathrm{kHz}$ in the range of frequencies where the type (A) has a pass band (Fig. 12).

Figure 15 displays the overall effect on the frequency response of a periodic shell with 4 consecutive type (B) cells. Although the first natural frequency is not affected by this configuration, all peaks between $2 \mathrm{kHz}$ and $3.5 \mathrm{kHz}$ vanish.

The bi-periodic configuration (C) allows for attempting a combination of the effects shown in the two previous cases. The bi-periodic cell consists of three sub-elements with the same taper ratio followed by one with opposed taper ratio.

The characteristics are displayed in Fig. 16. The principal stop band at low frequencies that was observed in type (A) characteristics (Fig. 12) is combined with the mid-frequencies stop band similar to the one of Fig. 13 (type (B) element).

The overall frequency response of a periodic shell with 2 consecutive bi-periodic cells appears in Fig. 17. Only disturbance about $3 \mathrm{kHz}$ can effectively propagate along the shell.

\section{Conclusions}

In this work, we developed a theoretical method based on the Transfer Matrix Formulation and the Wavelet Transforms that effectively simulates the influence of periodicity, variable geometry and material properties on the wave propagation characteristics of axis-symmetric shells. Several experiments were carried out in order to verify 

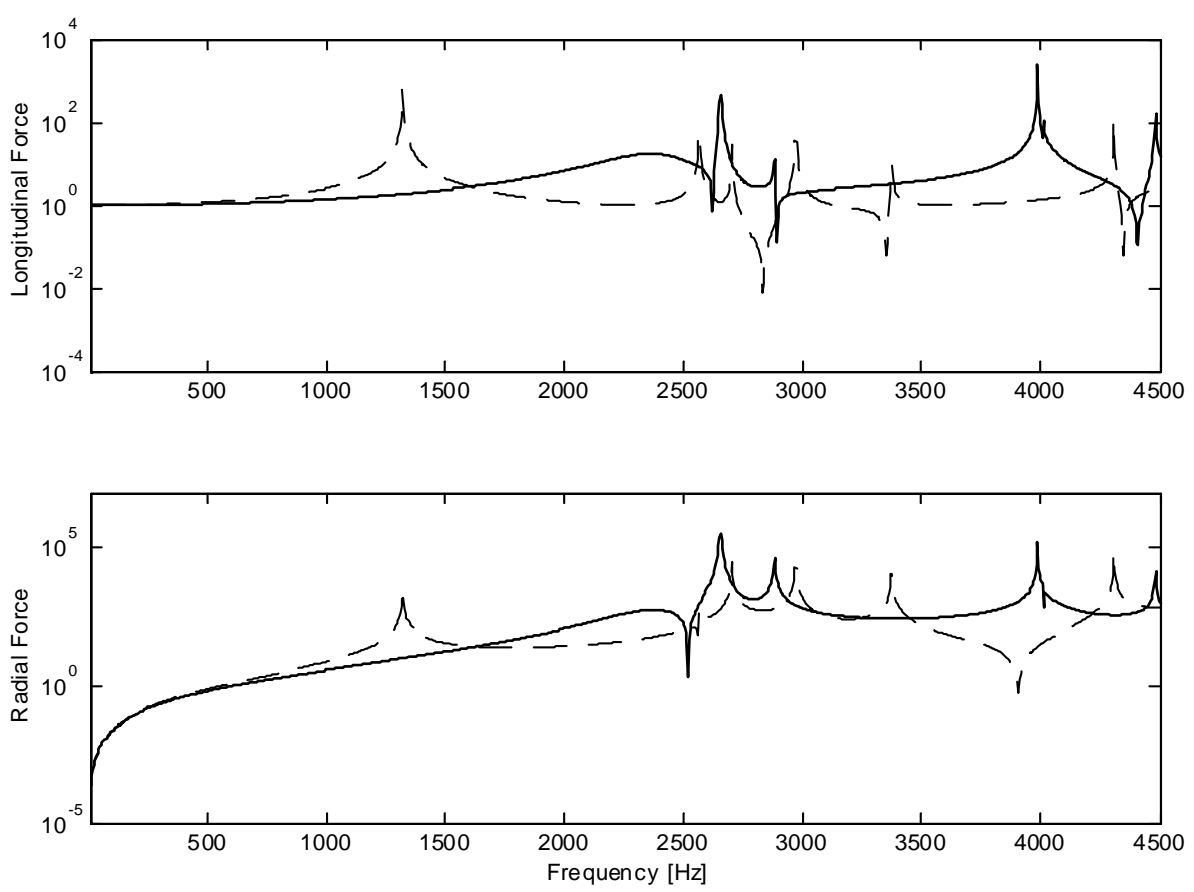

Fig. 13. Transmitted force: type (A) tapered shell (solid), uniform shell (dashed).
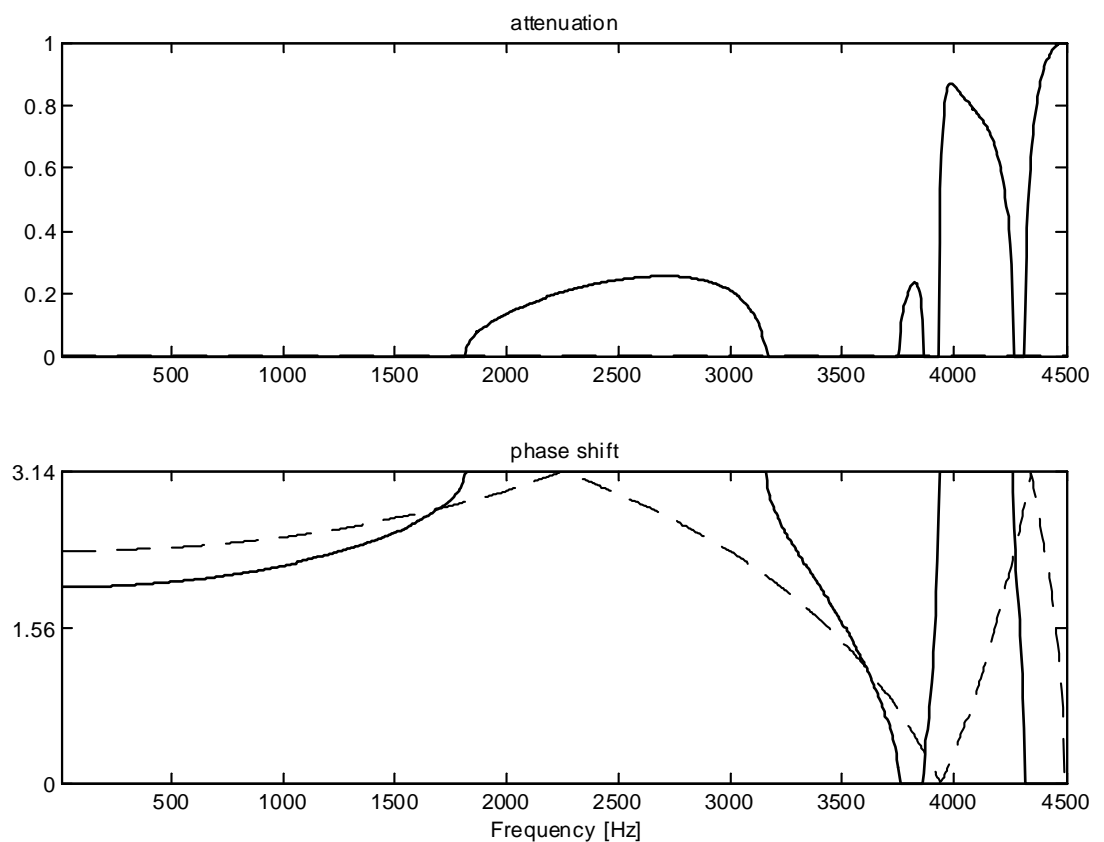

Fig. 14. Propagation characteristics of a periodic tapered shell type (B).

the numerical predictions. It is shown also that the Wavelet Transform has proven to be very powerful tool to uniquely identifying and comparing the energy distribution both in the time-frequency domain.

Thin shells can be modeled as two-dimensional wave-guides, where the propagation of the longitudinal waves is coupled with the flexural (radial) waves. A much richer scenario comes out as a result of this. Variations of the wall 

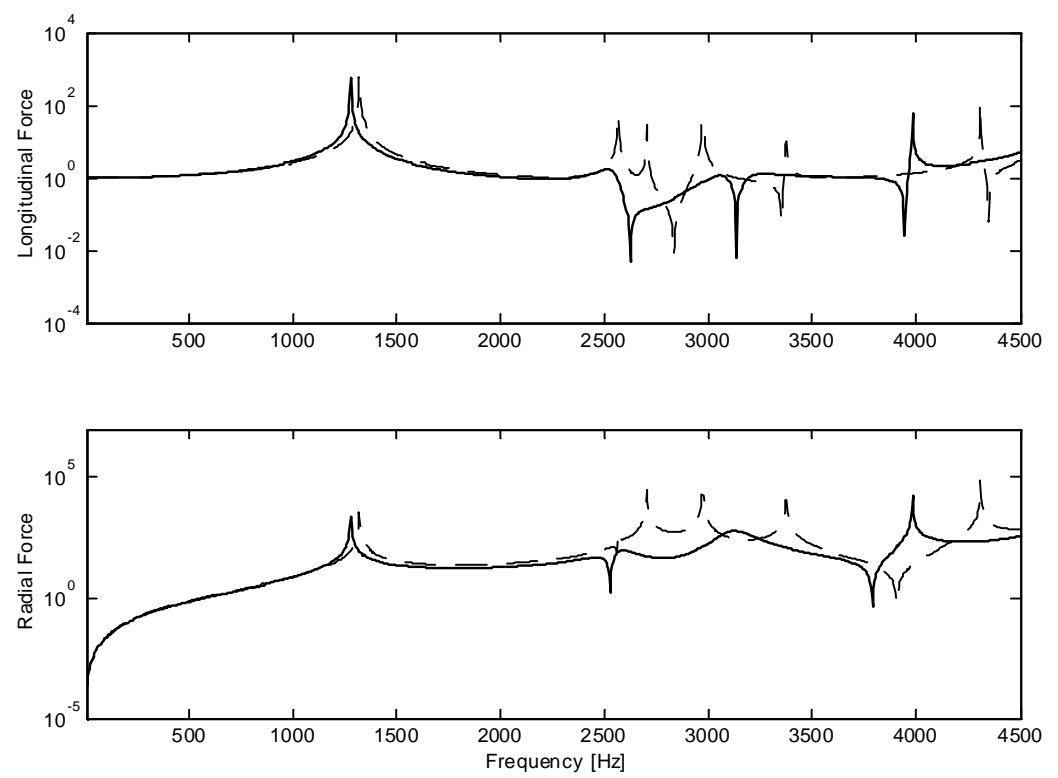

Fig. 15. Transmitted force: type (B) tapered shell (solid), uniform shell (dashed).
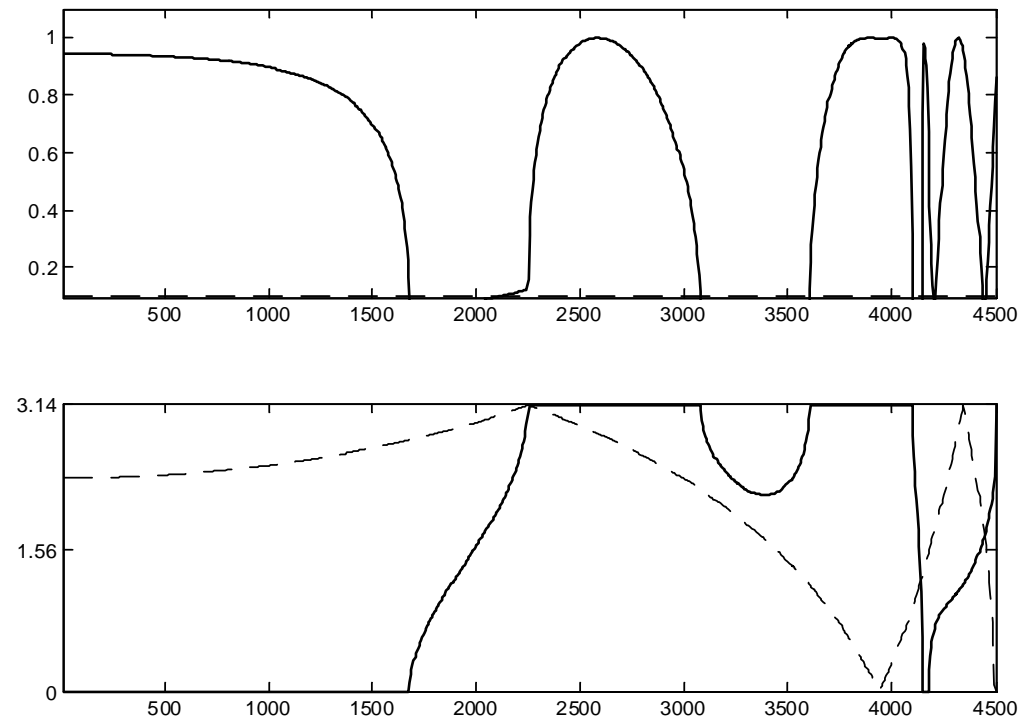

Fig. 16. Propagation characteristics of a periodic tapered shell type (C).

thickness, medium radius and element length of the shell can effectively filter out undesirable bands of frequencies from the longitudinal and/or the transverse wave patterns. Still, the principal parameter that influences the width of the stop bands is the ratio between the cross sections at the two ends of the shell element.

It was also shown that there are no significant improvements in the propagation characteristics when exponential profiles were implemented instead of simpler linear polynomials.

Very similar results were obtained with functionally graded materials. Materials like that can be employed in applications where the geometry of the structure is a constraint from a design point of view. Besides, it may be easier to obtain much higher ratios. Finally, by combining the two effects one can obtain the flexibility needed for some very demanding applications.

Different types of periodic taper configurations proved to have complementary effects on the wave character- 

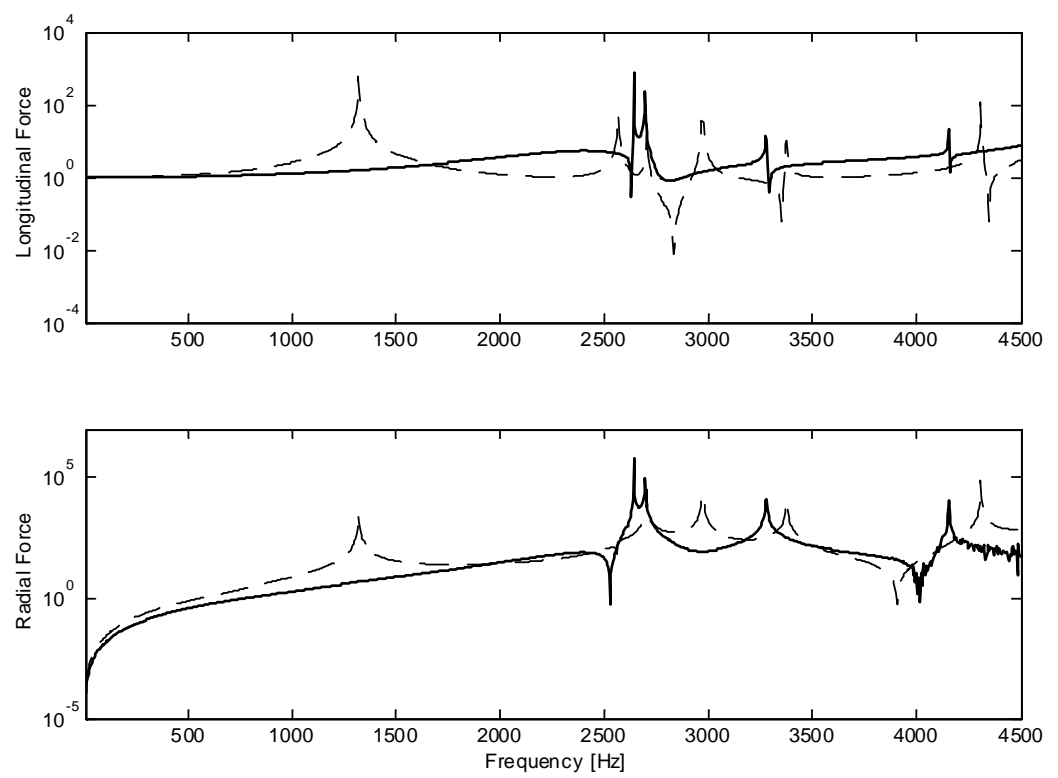

Fig. 17. Transmitted force: type (C) tapered shell (solid), uniform shell (dashed).

istics. Combinations of these complex geometries (bi-periodic tapered cells) produce the most effectual energy redistribution.

With the analytical frame work presented in this paper, it would be logical to augment such a frame work with appropriate optimization strategies to synthesize the shape and material distribution along the shell in a rational manner. Furthermore, development of the solutions of the inverse problem for shape and material distribution would also be a natural extension of the present study. In both the optimization and inverse problems, emphasis could be placed on targeting certain frequency bands where dominate vibration may occur.

\section{Appendix A: The wavelet transform}

The Wavelet Transform (WT) of a signal $x(t)$ is an example of a time-scale decomposition obtained by dilating and translating along the time axis a chosen analyzing function (wavelet) [1]. The integral or continuous WT relative to some basic wavelet $\psi(t)$ is defined as:

$$
W_{\psi}(a, b)=\frac{1}{\sqrt{a}} \int_{-\infty}^{+\infty} x(t) \cdot \psi^{*}\left(\frac{t-b}{a}\right) d t
$$

where $b$ is a translation parameter used for positioning the function $\psi(t)$ over the time domain, and $a>0$ is a scaling parameter dilating or contracting the function $\psi(t)$. The WT provides a flexible time-frequency window, which automatically narrows when observing high frequency phenomena and widens when studying low frequency components [1]. The wavelet function used in this paper is the Morlet wavelet, defined in the time domain as:

$$
\psi(t)=e^{-\frac{t^{2}}{2}} \cdot e^{j \cdot \omega_{w} \cdot t}
$$

The Morlet wavelet is a sinusoidal function, oscillating at the frequency $\omega_{w}$, modulated by a gaussian envelope of unit variance. Being composed of a modulated sinusoidal function, the Morlet wavelet is well suited for reproducing and analyzing signals in many applications and particularly in this work.

As signal decomposition, the WT cannot be directly compared to a time-frequency representation. However, it can be shown that $\mathrm{b}$ represents a time parameter and that the dilation parameter a is strictly related to frequency [11, 13]. In the frequency domain, the Morlet wavelet becomes: 


$$
\Psi(\omega)=\sqrt{2 \cdot \pi} \cdot e^{-\frac{1}{2} \cdot\left(\omega-\omega_{w}\right)^{2}}
$$

Equation (A3) shows that the frequency domain formulation of the Morlet wavelet is a gaussian function centered at $\omega=\omega_{w}$. Its dilated version is expressed as:

$$
\Psi(a \cdot \omega)=\sqrt{2 \cdot \pi} \cdot e^{-\frac{1}{2} \cdot\left(a \cdot \omega-\omega_{w}\right)^{2}}
$$

whose maximum is located at $a \cdot \omega=\omega_{w}$. Since $\omega_{w}=1.875 \pi$ is a fixed parameter defining the wavelet function [11], the center of the gaussian curve and therefore the frequency of the analysis can be located by changing the dilation parameter as follows:

$$
\omega=\frac{\omega_{w}}{a}
$$

The scale parameter can be hence considered as the inverse of a frequency parameter and thus the WT can be classified as a time-frequency transform.

An alternative formulation of the continuous WT can be obtained transforming both the signal $x(t)$ and the wavelet function $\psi(t)$ in the frequency domain:

$$
W_{g}(a, b)=\sqrt{a} \cdot \int_{-\infty}^{+\infty} X(\omega) \cdot \Psi^{*}(a \cdot \omega) \cdot e^{j \cdot \omega \cdot b} \cdot d \omega
$$

being $X(\omega)$ and $\Psi^{*}(a \omega) \cdot e^{j \cdot \omega_{0} \cdot b}$ the Fourier transforms of $x(t)$ and $\Psi^{*}\left(\frac{t-b}{a}\right)$ respectively.

This formulation of the WT can be expressed in a discrete form as:

$$
W(m, n) \sqrt{m \cdot \Delta a} \sum_{n} X\left(f_{n}\right) \cdot \Psi^{*}\left(m \cdot \Delta a \cdot f_{n}\right) \cdot e^{j \cdot 2 \cdot \pi \cdot f_{n} \cdot n \cdot \Delta b}
$$

where $f_{n}$ is the discrete frequency and $\Delta a$ and $\Delta b$ are discrete increments of dilation and translation parameters. Equation (A.7) allows an easy implementation of the WT. The frequency domain formulation of the WT is particularly convenient when the signal to be analyzed is expressed in the frequency domain.

\section{Nomenclature}

$\begin{array}{ll}A & \text { Area } \\ \text { A } & \text { State-space matrix } \\ c & \text { Characteristic wave speed } \\ D_{o} & \text { Outer diameter } \\ D_{t} & \text { Transverse elastic coefficient } \\ E & \text { Young's modulus of elasticity } \\ g, q & \text { Longitudinal and transverse rigidity } \\ G & \text { Transformation matrix } \\ h & \text { Shell thickness } \\ I & \text { Identity matrix } \\ k & \text { Wave number } \\ K_{t} & \text { Longitudinal elastic coefficient } \\ M & \text { Bending moment } \\ m_{b}, m_{D} & \text { Element bending and electric mass matrices respectively } \\ N & \text { Longitudinal traction force } \\ Q & \text { Shear force } \\ R, r & \text { Shell radius }\end{array}$




$\begin{array}{ll}T & \text { Kinetic energy } \\ \mathbf{T} & \text { Trasfer matrix } \\ U & \text { Potential energy } \\ U, u & \text { Longitudinal displacement } \\ V & \text { Volume } \\ V, W, v, w & \text { Transverse displacement } \\ W & \text { External work } \\ w_{b}, w_{D} & \text { Nodal transverse and electric displacements respectively } \\ Y & \text { Displacement vector } \\ Z & \text { State-space vector } \\ \alpha & \text { Propagation constant amplitude } \\ \beta & \text { Propagation constant phase angle } \\ \vartheta & \text { Angular coordinate } \\ \partial(\cdot) & \text { Partial derivative } \\ \delta(\cdot) & \text { First variation } \\ \varepsilon & \text { Strain } \\ \gamma & \text { Shear strain } \\ \Lambda & \text { Eigenvalues matrix of the transfer matrix } \\ \mu & \text { Propagation constant } \\ \rho & \text { Mass density } \\ \sigma & \text { Stress } \\ v & \text { Poisson's ratio } \\ \omega, \Omega & \text { Frequency } \\ S u b s c r i p t s & \\ D & \text { Related to electric degrees of freedom } \\ L & \text { At location L } \\ N & \text { N elements } \\ 0 & \text { At location } 0 \\ r, \vartheta & \text { In the } r, \vartheta \text { direction } \\ u & \text { Relative to the uniform profile } \\ x y z & \text { In the } x y z \text { direction } \\ , x & \text { Derivative in the x-direction } \\ y & \text { Derivative in the y-direction } \\ -1 & \text { Matrix transpose } \\ & \end{array}$

\section{References}

[1] C.K. Chui, Wavelets Analysis and Applications: An Introduction to Wavelets, Vol. 1, Academic Press Inc., 1992.

[2] W. Flugge, Stresses in Shells, Springer-Verlag, Berlin, 1973.

[3] K.F. Graff, Wave Motion in Elastic Solids, Dover Publications, New York, 1975.

[4] R.P.S. Han and J.D. Liu, Free Vibration Analysis of a Fluid-Loaded Variable Thickness Cylindrical Tank, Journal of Sound and Vibration 176(2) (1994), 235-253.

[5] T. Irie, G. Yamada and Y. Kaneko, Free Vibration of a Conical Shell with Variable Thickness, Journal of Sound and Vibration 82 (1982), 83-94.

[6] J.H. Kang and A.W. Leissa, Three-Dimensional Vibration of Hollow Cones and Cylinders with Linear Thickness Variations, Journal of Acoustical Society of America 106(2) (1999), 748-755.

[7] H. Kolsky, Stress waves in solids, Dover Publications, New York, 1963.

[8] A.W. Leissa and J. So, Three-Dimensional Vibration of Hollow Cones and Cylinders with Linear Thickness Variations, Journal of Acoustical Sa 106(2) (1999), 748-755.

[9] A.W. Leissa, Vibrations of Shells, (NASA SO-288), U.S. Government Printing Office, Washington D.C., 1973. 
[10] L. Meirovich, Principles and Techniques of Vibrations, Prentice Hall, New Jersey, 1997.

[11] T. Onsay and A.G. Haddow, Wavelet Transform Analysis of Transient Wave Propagation in a Dispersive Medium, Journal of Acoustical Society of America 95(3) (1994), 1441-1449.

[12] L.E. Penzes and J. Padovan, Maxi-Element Analysis for Free Vibration of Orthotropic Shells of Revolution, Journal of Sound and Vibration 54(2) (1977), 249-26.

[13] G. Solaroli, Z. Gu, A. Baz and M. Ruzzene, Wavelet Analysis of Wave Propagation in Shells with Periodic Stiffeners, Journal of Vibration and Control 9(9) (2003), 1057-1081.

[14] K.R. Sivadas and N. Ganesan, Axisymmetric Vibration Analysis of Thick Cylindrical Shell with Variable Thickness, Journal of Sound and Vibration 160(3) (1993), 387-400.

[15] K.R. Sivadas and N. Ganesan, Free Vibratio ylindrical Shells with Axially Varying Thickness, Journal of Sound and Vibration 147(1) (1991), 73-85.

[16] J.E. Stoneking, Free Vibrations of Shells of Revolution with Variable Thickness, Journal of Nuclear Engineering and Design 24 (1973), 314-321.

[17] K. Suzuki, T. Kosawada, G. Shikanai and K. Hayashi, Vibrations of Rotating Circular Cylindrical Shell with Varying Thickness, Journal of Sound and Vibration 166(2) (1993), 267-282.

[18] K. Suzuki, S. Takahashi, E. Anzai and T. Kosawada, Vibration of a Cylindrical Shell with Variable Thickness Capped by a Circular Plate, JSME 26(220) (1983), 1775-1782.

[19] S. Takahashi, K. Suzuki and T. Kosawada, Vibrations of a Conical Shells with Variable Thickness, JSME 29 (1986), $4306-4311$.

[20] S. Takahashi, K. Suzuki, E. Anzai and T. Kosawada, Axisymmetric Vibrations of a Conical Shells with Variable Thickness, JSME 25 (1982), 1771-1780.

[21] G.Y. Wang, Vibration of Building and Structures, Science and Technology Press, Beijing, 1998. 

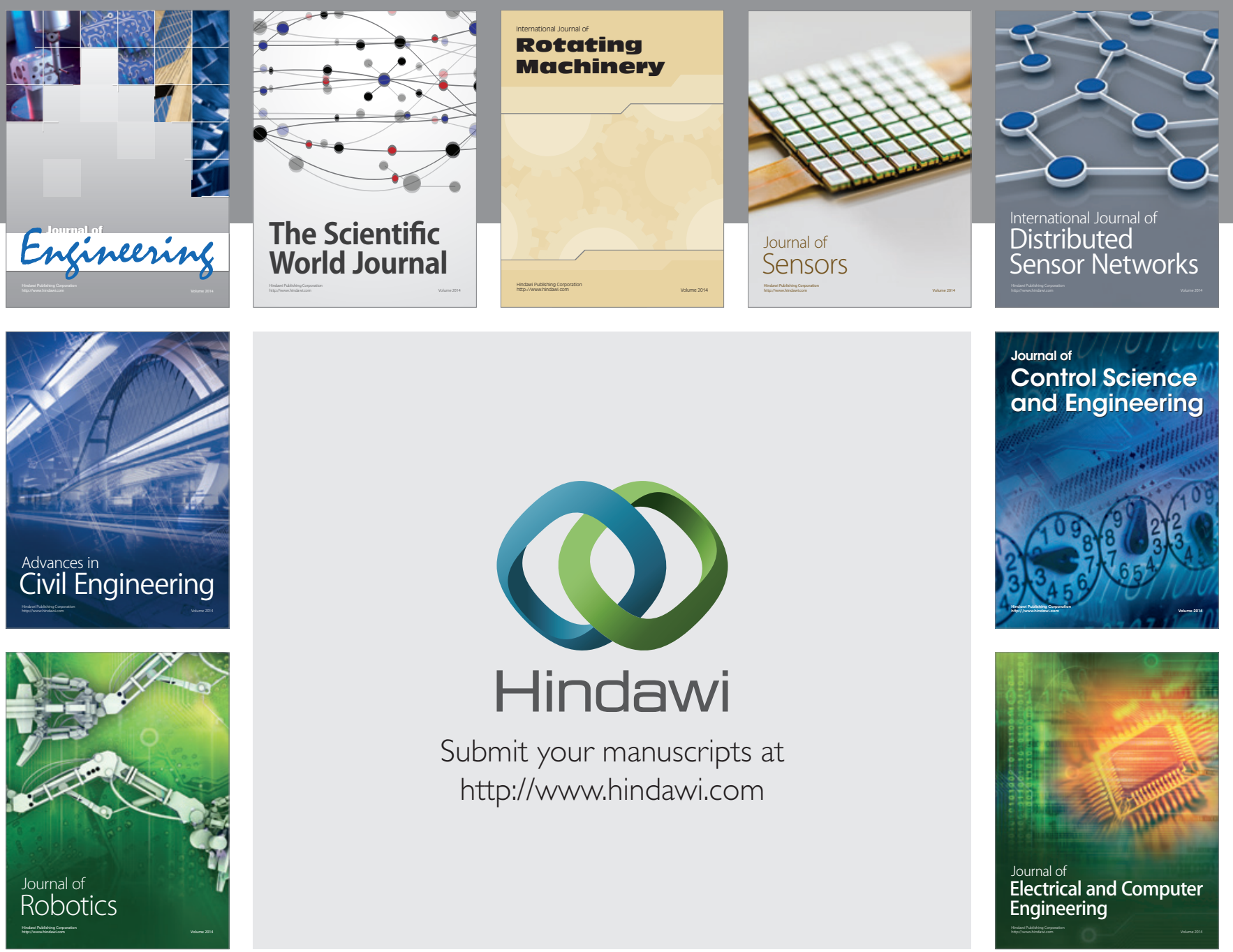

Submit your manuscripts at

http://www.hindawi.com
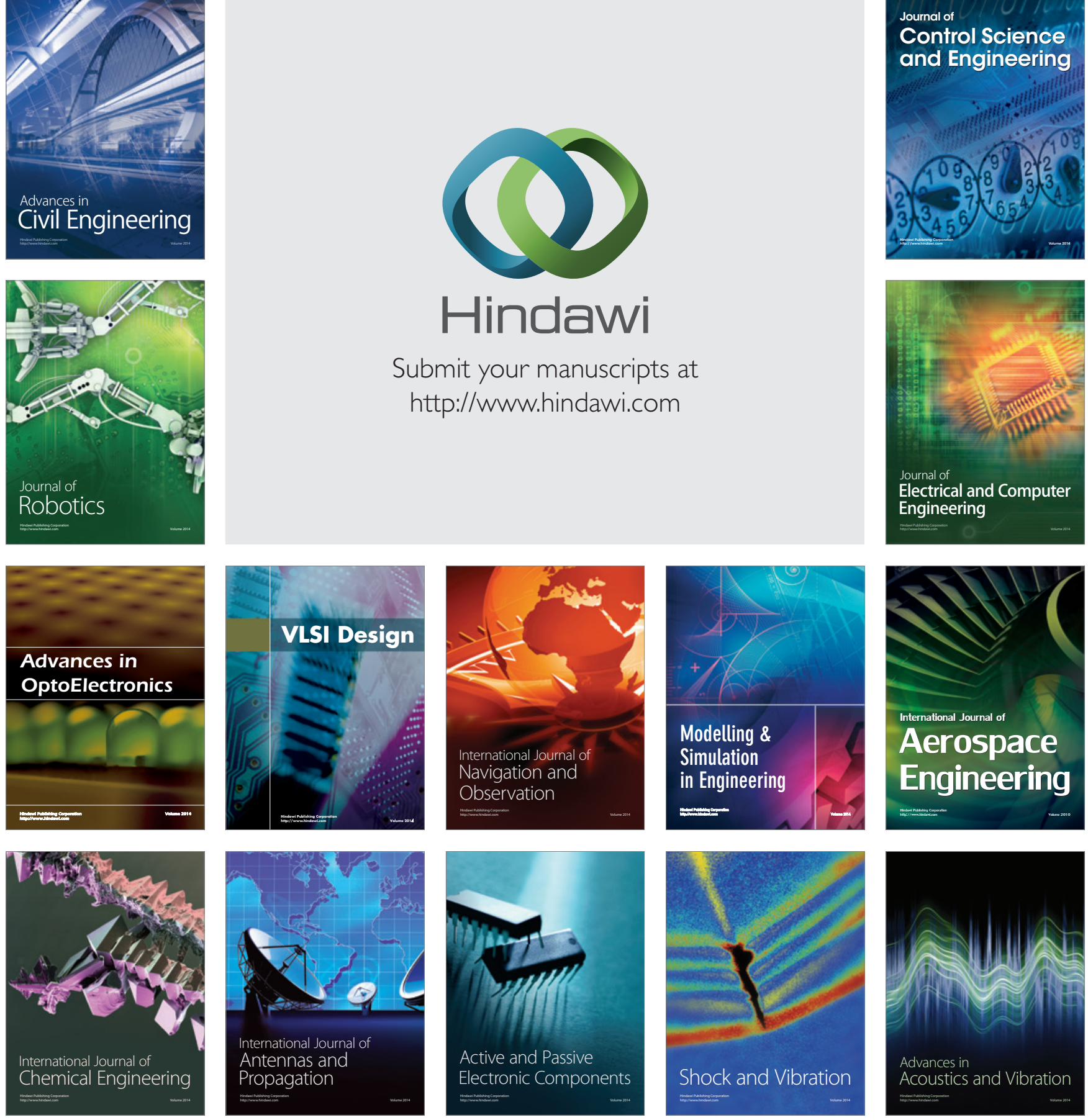